\title{
Neoantigen identification strategies enable personalized immunotherapy in refractory solid tumors
}

\author{
Fangjun Chen, Zhengyun Zou, Juan Du, Shu Su, Jie Shao, Fanyan Meng, Ju Yang, Qiuping Xu, Naiqing Ding, Yang Yang, Qin Liu, \\ Qin Wang, Zhichen Sun, Shujuan Zhou, Shiyao Du, Jia Wei, and Baorui Liu \\ The Comprehensive Cancer Centre of Drum Tower Hospital, Medical School of Nanjing University and Clinical Cancer Institute of Nanjing University, Nanjing, China.
}

\begin{abstract}
BACKGROUND. Recent genomic and bioinformatic technological advances have made it possible to dissect the immune response to personalized neoantigens encoded by tumor-specific mutations. However, timely and efficient identification of neoantigens is still a major obstacle to personalized neoantigen-based cancer immunotherapy.
\end{abstract}

\begin{abstract}
METHODS. Two different pipelines of neoantigen identification were established in this study: (a) Clinical-grade targeted sequencing was performed in patients with refractory solid tumor, and mutant peptides with high variant allele frequency and predicted high HLA-binding affinity were synthesized de novo. (b) An inventory-shared neoantigen peptide library of common solid tumors was constructed, and patients' hotspot mutations were matched to the neoantigen peptide library. The candidate neoepitopes were identified by recalling memory T cell responses in vitro. Subsequently, neoantigen-loaded dendritic cell vaccines and neoantigen-reactive T cells were generated for personalized immunotherapy in 6 patients.
\end{abstract}

\begin{abstract}
RESULTS. Immunogenic neoepitopes were recognized by autologous T cells in 3 of 4 patients who used the de novo synthesis mode and in 6 of 13 patients who used the shared neoantigen peptide library. A metastatic thymoma patient achieved a complete and durable response beyond 29 months after treatment. Immune-related partial response was observed in another patient with metastatic pancreatic cancer. The remaining 4 patients achieved prolonged stabilization of disease with a median progression-free survival of 8.6 months.
\end{abstract}

CONCLUSION. The current study provides feasible pipelines for neoantigen identification. Implementing these strategies to individually tailor neoantigens could facilitate neoantigen-based translational immunotherapy research.

TRIAL REGISTRATION. ChiCTR.org ChiCTR-OIC-16010092, ChiCTR-OIC-17011275, ChiCTR-0IC-17011913; ClinicalTrials.gov NCT03171220.

FUNDING. This work was funded by grants from the National Key Research and Development Program of China (2017YFC1308900), the National Major Projects for “Major New Drugs Innovation and Development" (2018ZX09301048-003), the National Natural Science Foundation of China (81672367, 81572329, 81572601), and the Key Research and Development Program of Jiangsu Province (BE2017607).

\section{Introduction}

T cell-based immunotherapy has been successfully used to treat many human solid cancers (1). Administering autologous tumorinfiltrating $\mathrm{T}$ lymphocytes (TILs) can lead to complete, durable tumor regressions in patients with metastatic melanoma (2). Meanwhile, checkpoint blockade immunotherapies have shown quite remarkable clinical responses in patients with advanced nonsmall cell lung cancer, melanoma, bladder cancer, gastric cancer, and colorectal cancers with DNA mismatch repair deficiency (3-7). Recently, increasing evidence has shown that $\mathrm{T}$ cells specific for neoepitopes (neoantigens), which are derived from mutated gene

Authorship note: FC and ZZ are co-first authors.

Conflict of interest: The authors have declared that no conflict of interest exists. Copyright: @ 2019, American Society for Clinical Investigation.

Submitted: December 28, 2017; Accepted: February 26, 2019; Published: April 8, 2019 Reference information: / Clin Invest. 2019;129(5):2056-2070.

https://doi.org/10.1172/JCI99538. products, are responsible for tumor regression in patients receiving TIL therapy $(8,9)$, and immune checkpoint inhibitor therapy in both mouse models and clinical settings $(7,10-12)$. By the de novo generation that is derived from tumor-specific somatic mutations, neoantigen-specific $\mathrm{T}$ cells are not subject to central and peripheral tolerance and also lack the ability to induce normal tissue destruction. Thus, neoantigens appear to represent ideal targets for $\mathrm{T}$ cell-based cancer immunotherapy. Strategies that harness a $\mathrm{T}$ cell response against neoantigens may be of significant clinical benefit in cancer patients.

Neoantigens promise high specificity but are largely patient-specific and, therefore, are hard to identify and are mainly singular events in a patient cohort. The classical cDNA library screening approach is labor-intensive, low-throughput, and incapable of identifying some mutated antigens derived from GC-rich transcripts and low-expression transcripts $(13,14)$. Nevertheless, recent technological advances in next-generation sequencing and bioinformatics analysis have provided a strong foundation on which to build these 
efforts. A peptide-based screening approach involving whole-exome sequencing (WES) and MHC-peptide binding prediction algorithms has been successful in identifying neoantigens recognized by TILs in patients with melanoma (9). Furthermore, use of tandem minigenes (TMGs) composed of multiple minigenes that encode polypeptides containing a mutated amino acid residue flanked on their $\mathrm{N}$ - and C-termini by $12-13$ amino acids, which were synthesized and used to transfect antigen-presenting cells (APCs), has led to success in identifying neoantigens in patients with melanoma and cholangiocarcinoma and murine tumor models (15-17).

Recent methods of immunogenic neoantigen identification are often required to synthesize dozens to hundreds of peptides, but are time-consuming and costly and have low positive rates. Even when a series of TMGs is constructed, further synthesis of peptides is needed to verify the bona fide neoepitope harbored in the immunogenic TMG. Thus, narrowing down the list of potential neoepitopes and reducing the time of the identification process are currently major unresolved clinical challenges, particularly for highly mutated and advanced refractory cancers. In addition, whole-genome sequencing, WES, and transcriptome sequencing, which are performed mainly in neoantigen identification at present, are not suitable for liquid biopsy samples that require extremely high sequencing depths (no less than $3000 \times$ ). Moreover, recurrent hotspot mutations of the driver gene could overcome the problem of patient specificity and could be targeted in broadly applicable immunotherapeutic treatments of different types of cancers. Indeed, $\mathrm{T}$ cells that recognize BCR-ABL, mutant IDH1-R132H, and KRAS-G12D have been identified, and vaccines and adoptive $\mathrm{T}$ cell therapy against these mutations have shown promise in preclinical and clinical studies (18-20). However, systematic immunogenicity assessment of neoepitopes for common driver mutations in solid tumors is currently lacking.

Considering the aforementioned limitations, 2 different pipelines for rapid and efficient personalized neoantigen were identified in patients with an advanced solid tumor. Clinical-grade targeted genomic profiling of tumor, circulating tumor DNA (ctDNA), and matched normal samples was performed to identify nonsynonymous somatic mutations. As the first mode, somatic mutations were subjected to in silico analysis to predict and prioritize potential high-affinity epitopes, and then mutated peptides were synthesized de novo accordingly. In addition, an inventoryshared driver mutation-derived neoantigen peptide library was constructed by systematic mining of The Cancer Genome Atlas (TCGA) and Catalogue of Somatic Mutations in Cancer (COSMIC) databases and use of multiple epitope prediction programs. Patients' recurrent hotspot mutations were matched to the customized neoantigen peptide library. The candidate mutated peptides in different pipelines were screened to identify T cell neoepitopes for recognition by autologous peripheral blood mononuclear cells (PBMCs) in vitro. Moreover, personalized neoantigenpulsed dendritic cell (DC) vaccines and neoantigen-reactive T cell (NRT) adoptive transfer immunotherapy were performed to evaluate the safety and antitumor efficacy.

\section{Results}

Targeted sequencing-guided neoantigen identification by the peptide de novo synthesis model. Personalized neoantigen identification was performed in vitro on 4 patients with advanced solid tumor who underwent 416-gene panel sequencing and HLA typing based on PCR-sequence-based typing (PCR-SBT) (Supplemental Table 1; supplemental material available online with this article; https://doi.org/10.1172/JCI99538DS1). Somatic mutations with allele frequency (AF) greater than $2 \%$ were selected to predict $\mathrm{T}$ cell epitopes that bind to patients' HLA class I and class II allotypes (Supplemental Table 2). Specifically, NetMHC 3.4/NetMHC 4.0 and NetMHCpan 3.0 were used to predict MHC class I-restricted T cell epitopes, and NetMHCII 2.2 was used to predict MHC class II-restricted $\mathrm{T}$ cell epitopes. The predicted neoepitopes were ranked, and peptides were prioritized according to the following criteria: (a) strong binders with $\mathrm{IC}_{50}$ less than $50 \mathrm{nM}$ or a percentage rank of affinity (\%rank) of less than 0.5; (b) peptides with mutations with higher tumor variant allele frequency; (c) peptides predicted to bind 2 or more HLA molecules; and (d) peptides that could be predicted by different algorithms. To further characterize the specificity of the preexisting $\mathrm{T}$ cell response to the prioritized mutant peptides, each patient's PBMCs were stimulated with peptides for 10 days in the presence of IL-2. Subsequently, both the secretion of the effector cytokine IFN- $\gamma$ using an enzyme-linked immunospot (ELISPOT) assay and the upregulation of the $\mathrm{T}$ cell activation marker 4-1BB using flow cytometry were measured, since these approaches could provide complementary and nonredundant information about antigen-specific $\mathrm{T}$ cell responses.

First, neoantigen identification was performed based on the somatic nonsynonymous mutation in tumor samples of 2 patients (ID: A008, A017). Patient A008 with metastatic pancreatic cancer was enrolled, and the top 9 predicted binding peptides restricted by autologous MHC class I and class II allotypes were synthesized and tested for recognition by autologous PBMCs in vitro (Supplemental Table 3). The ELISPOT assay and flow cytometry analysis consistently demonstrated that an $\mathrm{A}^{\star} 3001$-restricted $\mathrm{CD} 8^{+} \mathrm{T}$ cell epitope (TP53-V25G-1, RGRAMAIYK) and a DRB1 ${ }^{*} 0701$-restricted CD4 ${ }^{+}$ $\mathrm{T}$ cell epitope (DIS3L2-I777V, MVMGVLKQAFDVLVL) induced significant peptide-specific $\mathrm{T}$ cell responses (Figure 1, A and B).

For patient A017 with metastatic thymoma, who expressed the highly prevalent HLA class I allele HLA-A ${ }^{*} 201$, three HLA-A ${ }^{*}$ O201-restricted $\mathrm{T}$ cell epitopes with the highest mutation abundance $(\mathrm{AF}>10 \%)$ and excellent binding affinity were selected, while incorporating nine $\mathrm{HLA}^{*} \mathrm{~A}^{*} \mathrm{O} 2$-restricted irrelevant mutant peptides from the customized shared neoepitope peptide library, to assess the $\mathrm{T}$ cell-specific antigen response level. The results demonstrated that the mutated CDC73-Q254E nonamer (NIFAILESV) stimulated high amounts of IFN- $\gamma$ spots and obvious $\mathrm{CD}^{+} 4-1 \mathrm{BB}^{+} \mathrm{T}$ cells, whereas no detectable responses were observed against the irrelevant mutant peptides or the control group (no peptide stimulation) (Figure 2, A-C, and Supplemental Tables 4 and 5). Subsequently, the binding affinity of the mutant CDC73 (CDC73-MT) and the corresponding wild-type (CDC73WT) peptides to $\mathrm{HLA}^{*} \mathrm{~A}^{*} 2 \mathrm{O} 1$ was assessed using the T2 cell line. The CDC73-MT peptide (NIFAILESV) showed substantial binding to HLA-A 0201 molecule, which was stronger than that of CDC73-WT (NIFAILQSV) at concentrations ranging from $6.25 \mu \mathrm{M}$ to $50 \mu \mathrm{M}$. Notably, though, the CDC73-WT peptide also showed a strong binding affinity at a higher concentration of 100 $\mu \mathrm{M}$ (Figure 2D and Supplemental Table 6). Thus, the specificity 


\section{Table 1. Alteration frequency of hotspot mutations in common solid tumors (TCGA)}

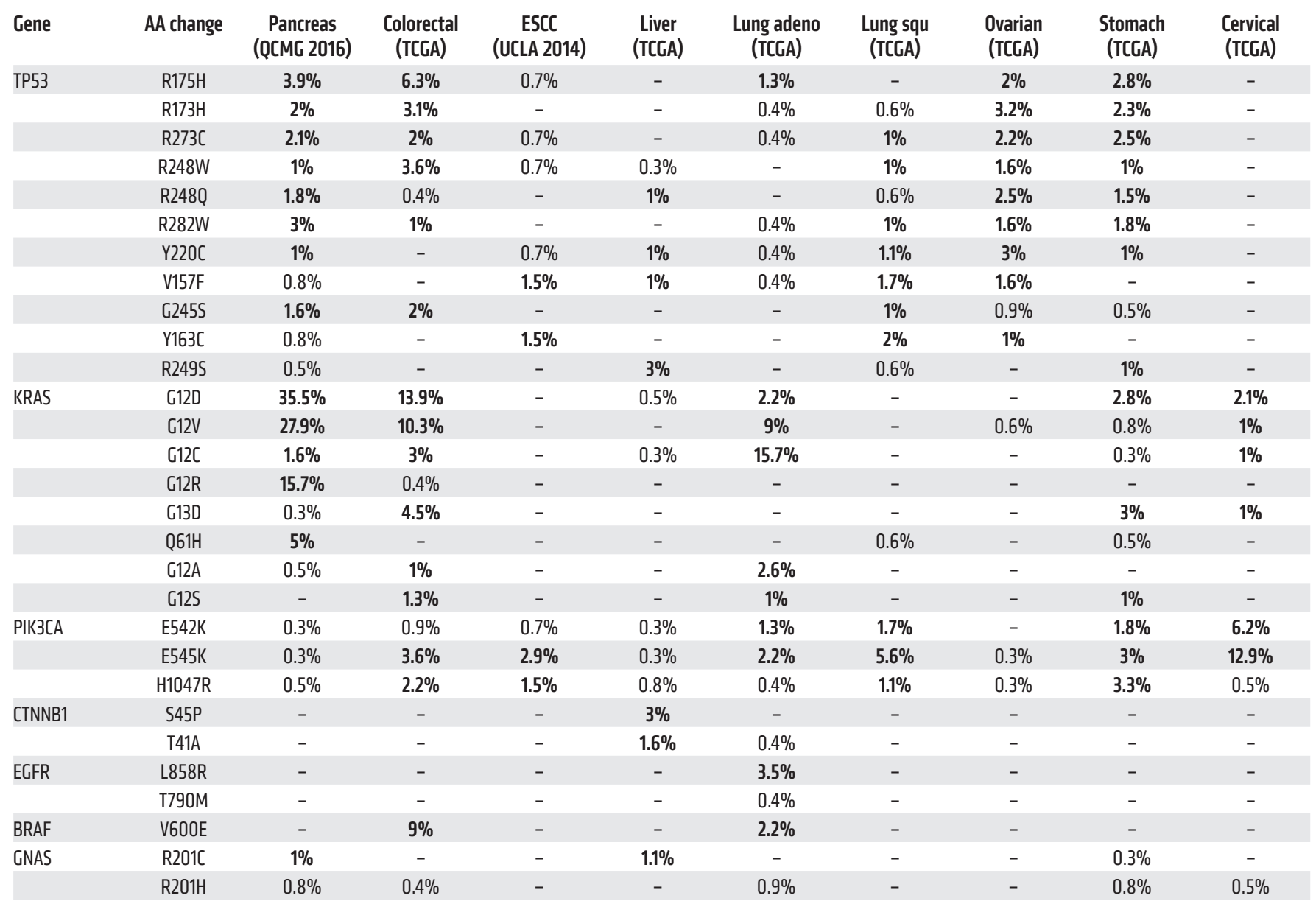

Alteration frequencies $\geq 1 \%$ are in boldface. QCMG, Queensland Centre for Medical Cenomics; ESCC, esophageal squamous-cell carcinoma; adeno, adenocarcinoma; squ, squamous cell.

and reactivity of autologous T cells against mutant and corresponding WT CDC73 peptides were further assessed at concentrations ranging from $0.01 \mathrm{nM}$ to $1000 \mathrm{nM}$. The patient's T cells recognized T2 cells pulsed with a minimum of $1.0 \mathrm{nM}$ of the mutated CDC73Q254E peptide but failed to recognize cells pulsed with $100 \mathrm{nM}$ of the corresponding WT peptides (Figure 2E). Even though the WT peptide showed moderate affinity to HLA-A ${ }^{*}$ 0201, it failed to induce the IFN- $\gamma$ secretion of autologous T cells, indicating that the mutated amino acids in the CDC73 peptide may predominantly affect $\mathrm{T}$ cell receptor contact residues. Subsequently, patient A017, who failed 3 lines of treatment, was enrolled in neoantigen-based personalized immunotherapy. Clinical-grade NRTs (bulk T cells composed of $\sim 7 \%$ neoantigen-reactive $\mathrm{CD} 8^{+} \mathrm{CD} 137^{+} \mathrm{T}$ cells; Supplemental Figure 1) showed a median of $39.5 \%$ specific killing of CDC73-Q254E peptide-loading T2 cells at an effector/target $(\mathrm{E} / \mathrm{T})$ ratio of $40: 1$ compared with a median of $17.2 \%$ of nonspecific cell lysis of unpulsed T2 cells. The specific lysis showed E/T ratio-dependent characteristics, with specific lysis decreasing with reduced $\mathrm{E} / \mathrm{T}$ ratios (Figure $2 \mathrm{~F}$ ).

Furthermore, neoantigen identification of the other 2 patients with advanced gastric cancer (ID: A004, A015) was based on somatic mutations that were present both in the tumor tissues and in plasma ctDNA. The nonsynonymous and frameshift mutations with AF greater than $2 \%$ were used to predict T cell epitopes that bound to each patient's HLA-A, HLA-B, and HLA-C alleles with a binding affinity (\%rank) less than 2.0. The final prioritized candidate mutant peptides were pursued for the immunogenicity study (Supplemental Tables 7 and 8). In patient A004, one neoepitope (CYP2A6-N438Y, KRYCFGEGL) of the 9 prioritized peptides, which was predicted to bind to both HLA-B ${ }^{*} 1402$ and HLA-C ${ }^{\star} 0704$, was recognized by autologous peripheral blood lymphocytes that were confirmed by IFN- $\gamma$ and $4-1 \mathrm{BB}$ expression levels (Figure 3, A and B). In patient A015, the prioritized 12 candidate mutant peptides were analyzed to repeatedly stimulate PBMCs in vitro, but no peptide-specific responses were detected (Supplemental Figure 2).

Assessment of mutational and neoantigen loads by targeted sequencing. To evaluate the potential of mutation and neoantigen identification, a large clinical-grade targeted sequencing panel of 416 cancer-related genes was performed in 17 patients with advanced solid tumor, and HLA typing data for each patient were determined by PCR-SBT (Supplemental Table 2). A median of 35 
A

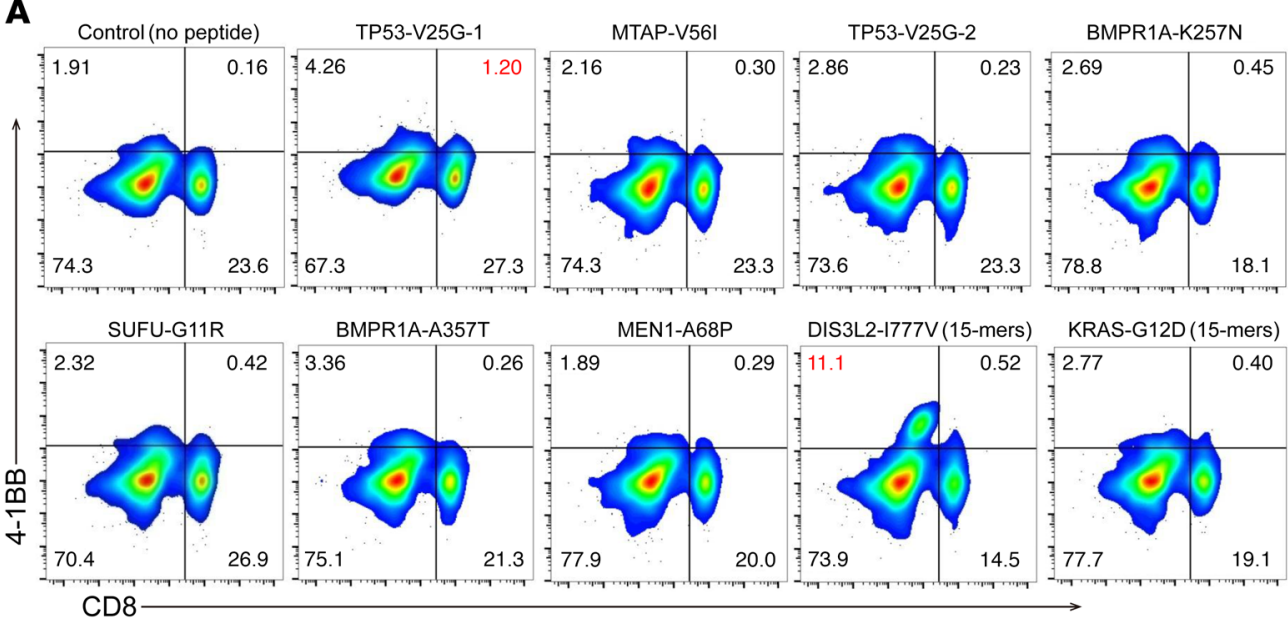

Figure 1. Identification of personalized neoantigen in patient $\mathbf{A 0 0 8}$ with metastatic pancreatic cancer. Autologous PBMCs were stimulated with candidate mutant peptides every 3 days in the presence of IL-2, and on day $10 \mathrm{~T}$ cell responses to each antigen were measured by flow cytometric analysis for 4-1BB upregulation on $\mathrm{CD} 8^{+} \mathrm{T}$ and $\mathrm{CD} 4^{+} \mathrm{T}$ cells (gated on CD3) (A) and IFN- $\gamma$ ELISPOT assay (B). The no-peptide (media) stimulation was tested as control. Data are representative of 3 independent experiments.

\section{B}

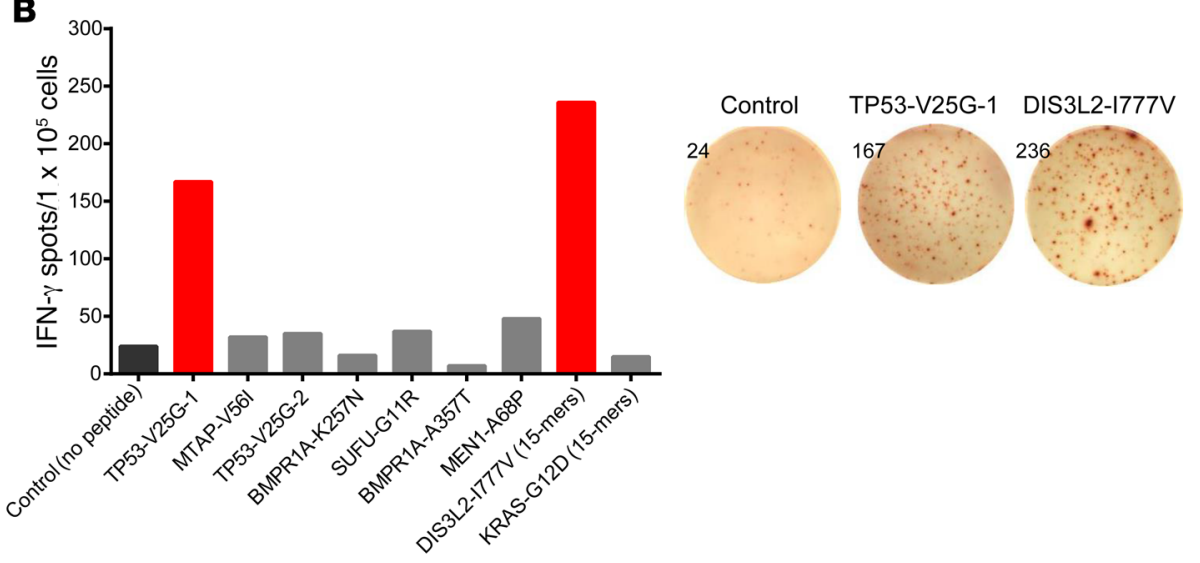

somatic missense mutations (range 9-73) was identified (Figure 4A). The candidate neoantigen epitopes were identified for each patient's nonsynonymous single-nucleotide variation mutations of the restricting HLA class I alleles (HLA-A, HLA-B, and HLA-C). A median of 55 predicted HLA class I-restricted neoantigens (range 8-140) were identified by the NetMHCpan 3.0 program with \%rank less than 2 . Among the aforementioned candidate epitopes, the number of strong binders (\%rank < 0.5$)$ ranged from 0 to 43 , with a median of 19 . Besides, the number of weak binders $(0.5<\%$ rank $<2)$ ranged from 8 to 98 , with a median of 44 (Figure $4 \mathrm{~B})$. The individual total number of mutations and complexity of HLA genotypes reflected the number of potential peptides that ranked within the cutoff criteria for testing. The 4 patients who performed neoantigen screening in vitro showed moderate and even more mutations among the 17 patients (Figure 4). Apparently, a large targeted sequencing panel has the potential to identify mutations and neoantigens for multiple patients with advanced solid tumors. Intriguingly, a frameshift mutation of the DNA polymerase epsilon (POLE) gene [p.V1446fs (c.4337_4338del)] was detected in the ctDNA of patient A017, who represented the highest mutational load among the 17 patients. Recently, Mehnert et al. revealed that mutation of POLE in patients with endometrial cancer was associated with an ultramutator phenotype beyond the microsatellite instability (MSI) phenotypes, as the presence of POLE mutation could detect $82.2 \pm 25$ somatic mutations using a panel of 315 cancer-related genes (FoundationOne, Foundation Medicine) (21).

Inventory-shared neoantigen peptide library construction. An off-shelf neoepitope peptide library was built with the aim of identifying neoantigens in a timely and convenient manner in refractory advanced solid tumors with a dismal prognosis. First, the TCGA and COSMIC databases were used to mine high-frequency mutant genes in 9 types of human malignant solid tumors, including gastric cancer, colorectal cancer, esophageal squamous cell carcinoma, liver cancer, lung adenocarcinoma, lung squamous cell carcinoma, pancreatic cancer, ovarian cancer, and cervical cancer, as well as to calculate the frequencies of the hotspot mutations in each gene by in silico analysis. A total of 21 mutant genes with frequency greater than $10 \%$ among the aforementioned solid tumors in the COSMIC database, which has the largest number of recorded samples in the world, were further evaluated in 2430 sequenced samples of the TCGA database (Supplemental Tables 9 and 10). Next, it was observed that the majority of the 21 recurrent mutant genes, in which missense mutations were dispersed throughout, could not serve as ideal shared antigen targets. However, 29 ideal hotspot mutations existed in KRAS, TP53, CTNNB1, EGFR, BRAF, PIK3CA, and GNAS (Table 1 and Supplemental Table 11), which were classified as cancer driver genes (22). Thus, the 29 hotspot mutations were selected as the candidate targets to build the shared neoantigen peptide library, which covered 
Table 2. Shared neoantigen peptide library construction of common solid tumors

\begin{tabular}{|c|c|c|c|}
\hline Gene & AA mutation & HLA type & Peptide $^{A}$ \\
\hline \multirow[t]{3}{*}{ CTNNB1 } & p.T41A & HLA-A1101 & ATAPSLSCK \\
\hline & p.T41A & HLA-A0203 & GIHSGATÁATA \\
\hline & p.S45P & HLA-A1101 & TTAPPLSGK \\
\hline \multirow[t]{3}{*}{ EGFR } & p.L858R & HLA-A1101 & KITDFGㅁAK \\
\hline & p.T790M & HLA-A0201/03/06 & MQQLMPFGCLL \\
\hline & p.T790M & HLA-A2402 & VQLIMQQLMPF \\
\hline GNAS & p.R201H & HLA-A0203 & LLRCHVLTS \\
\hline \multirow[t]{20}{*}{ KRAS } & p.G12D & HLA-A1101 & VVGADGVGK \\
\hline & p.G12D & HLA-A0201/03/06 & KLVVVGADEGV \\
\hline & p.G12D & HLA-A1101 & VVVGADEGGK \\
\hline & p.G13D & HLA-A1101 & VVGAGDVGK \\
\hline & p.G13D & HLA-A0201/03/06 & KLVVVGAGㅁV \\
\hline & p.G13D & HLA-A1101 & VVVGAGDVGK \\
\hline & p.G12V & HLA-A1101 & VVGAVGVGK \\
\hline & p.G12V & HLA-A0201/03/06 & KLVVVGAVGGV \\
\hline & p.G12V & HLA-A1101 & VVVGAVGGVGK \\
\hline & p.G12A & HLA-A1101 & VVGAEAGVGK \\
\hline & p.G12A & HLA-A0201/03/06 & KLVVVGAAGGV \\
\hline & p.G12A & HLA-A1101 & VVVGAAGGVKK \\
\hline & p.G12C & HLA-A1101 & VVGACGVGK \\
\hline & p.G12C & HLA-A0201/03/06 & KLVVVGACGCV \\
\hline & p.G12S & HLA-A1101 & VVGAS్GVGK \\
\hline & p.G12S & HLA-A0201/03/06 & KLVVVGASEGVV \\
\hline & p.G12S & HLA-A1101 & VVVGASSGVGK \\
\hline & p.G12R & HLA-A1101 & VVGARGVGK \\
\hline & p.G12R & HLA-A0201/03/06 & KLVVVGAR्RGV \\
\hline & p.G12R & HLA-A1101 & VVVGARGVCK \\
\hline РІКЗСА & p.E542K & HLA-A1101 & AISTRDPLSK \\
\hline \multirow[t]{12}{*}{ TP53 } & p.R248Q & HLA-A0203/06 & NQRPILTII \\
\hline & p.R248Q & HLA-A1101 & SSCMGGMNQR \\
\hline & p.R248Q & HLA-A0201/03 & GMNQRPILTI \\
\hline & p.R248W & HLA-A1101 & SSCMGGMNWWR \\
\hline & p.R248W & HLA-A0201/03 & CMNWWRPILTI \\
\hline & p.G245S & HLA-A1101 & SSCMGSMNR \\
\hline & p.G245S & HLA-A0201/03 & SMNRRPILTI \\
\hline & p.R249S & HLA-A0201/03 & GMNRS్PILTI \\
\hline & p.Y220c & HLA-A0201/06 & VVPCEEPPEV \\
\hline & p.V157F & HLA-A1101 & RERAMAIYK \\
\hline & p.V157F & HLA-A1101 & STPPPGTRER \\
\hline & p.Y163C & HLA-A1101 & RVRAMAICKK \\
\hline
\end{tabular}

\begin{tabular}{|c|c|c|}
\hline \multirow[b]{2}{*}{ NetMHC 4.0} & \multicolumn{2}{|c|}{ Predicted score } \\
\hline & BIMAS & NetCTL \\
\hline 13.9 & 1 & 1.2368 \\
\hline $83^{B}$ & - & - \\
\hline 16 & 1 & 1.3774 \\
\hline 162.8 & 0.12 & 0.9082 \\
\hline 28.7/42.8/19.9 & 51.77 & - \\
\hline 1385 (\%rank 1.4) & 3 & - \\
\hline 249 & - & 0.1541 \\
\hline 368.2 & 2 & 0.7525 \\
\hline 498/62/332.2 & 119.282 & - \\
\hline 430 & 3 & 0.755 \\
\hline 405.5 & 2 & 0.5986 \\
\hline $506.9 / 62 / 414.8$ & 31.646 & - \\
\hline 429.1 & 3 & 0.601 \\
\hline 65.5 & 2 & 1.065 \\
\hline $300.2 / 62 / 199.7$ & 243.432 & - \\
\hline 137.3 & 3 & 1.0674 \\
\hline 147.7 & 2 & 0.8661 \\
\hline $237.8 / 47.2 / 204.5$ & 243.432 & - \\
\hline 243.1 & 3 & 0.8685 \\
\hline 135 & 2 & 0.9417 \\
\hline $373.6 / 62 / 183.1$ & 243.432 & - \\
\hline 114.4 & 2 & 1.055 \\
\hline $390.7 / 33.8 / 338.4$ & 243.432 & - \\
\hline 213.1 & 3 & 1.0575 \\
\hline 163.1 & 2 & 1.0948 \\
\hline $506.9 / 61.5$ & 48.686 & - \\
\hline 414.8 & 3 & 1.0972 \\
\hline 50.8 & 0.8 & 1.1248 \\
\hline $68 / 269$ & - & 0.2897 \\
\hline 177 & 0.004 & - \\
\hline $444 / 30$ & 17.33 & - \\
\hline 169 & 0.004 & - \\
\hline $163 / 30$ & 17.33 & - \\
\hline 27 & 0.008 & 0.7161 \\
\hline $413 / 22$ & 17.33 & - \\
\hline $349 / 18$ & 17.33 & - \\
\hline $350 / 184$ & 10.346 & 0.8092 \\
\hline 256 & 1.2 & 1.298 \\
\hline 243 & 0.02 & - \\
\hline 65 & 12 & 1.3249 \\
\hline
\end{tabular}

\begin{tabular}{|c|c|}
\hline YFPEITHI & IEDB \\
\hline 25 & 0.3 \\
\hline 17 & - \\
\hline 22 & 0.4 \\
\hline 19 & 1.35 \\
\hline 12 & 3.85 \\
\hline 14 & 2.1 \\
\hline- & - \\
\hline 25 & 1.65 \\
\hline 22 & 3.45 \\
\hline 25 & 1.6 \\
\hline 25 & 1.9 \\
\hline 21 & 8.35 \\
\hline 25 & 1.65 \\
\hline 25 & 0.9 \\
\hline 24 & 2.15 \\
\hline 25 & 0.95 \\
\hline 25 & 1.35 \\
\hline 24 & 1.9 \\
\hline 25 & 1.2 \\
\hline 25 & 1.25 \\
\hline 22 & 2.35 \\
\hline 25 & 1 \\
\hline 22 & 2.6 \\
\hline 25 & 1 \\
\hline 25 & 1.3 \\
\hline 22 & 4 \\
\hline 25 & 1.3 \\
\hline 25 & 0.4 \\
\hline- & 5.45 \\
\hline 21 & 2.3 \\
\hline 25 & 4.35 \\
\hline 21 & 2.05 \\
\hline 25 & 2.85 \\
\hline 21 & 0.9 \\
\hline 26 & 3.95 \\
\hline 25 & 3.2 \\
\hline 18 & 4.5 \\
\hline 16 & 2.05 \\
\hline 22 & 2 \\
\hline 26 & 1.2 \\
\hline
\end{tabular}

${ }^{A}$ Mutated residues are underlined and in boldface. ${ }^{B} \mathrm{HLA}$-binding affinity of this peptide is predicted by NetMHC 3.4.

9.49\%-89.56\% of cancer patients in the TCGA database, with a median coverage of $23.04 \%$ (Figure $5 \mathrm{~A}$ ).

The design of 8- to 10-mer peptides that were predicted to bind to human high-frequency HLA-A class I gene products of subtypes HLA-A ${ }^{\star} 02\left(A^{\star} 0201, A^{*} 0203\right.$, and $\left.A^{*} 0206\right), H L A-A^{*} 11$ ( $\left.A^{*} 1101\right)$, and HLA-A $24\left(\mathrm{~A}^{*} 2402\right)$ was initiated using 19-mer peptides containing the mutated amino acid at position 10 with 5 programs using different algorithms: BIMAS, the Immune Epitope Database (IEDB), NetMHC 3.4/NetMHC 4.0, NetCTL 1.2, and SYFPEITHI. The design integrated prediction of peptide-MHC class I binding affinity, proteasomal C-terminal cleavage, transporter associated with antigen processing (TAP) transport efficiency, and half-time of dissociation of peptide-HLA class I molecules. Results from the epitope prediction analyses were ranked, with NetMHC 4.0/ NetMHC $3.4\left(\mathrm{IC}_{50}<500 \mathrm{nM}\right)$ as the primary tool plus support from other programs. The prioritized 44 shared neoepitope peptides were selected for peptide synthesis, and then lyophilized peptide powder was stored in aliquots at $-80^{\circ} \mathrm{C}$ until use (Table 2). Although a minority of hotspot mutations were predicted to lack binding affinity to the selected HLA class I alleles, the shared neoantigen library could still cover $5.11 \%-83.8 \%$ of patients in the 9 types of common solid tumors, with a median coverage of $11.2 \%$ (Figure $5 \mathrm{~B}$ ). 
A

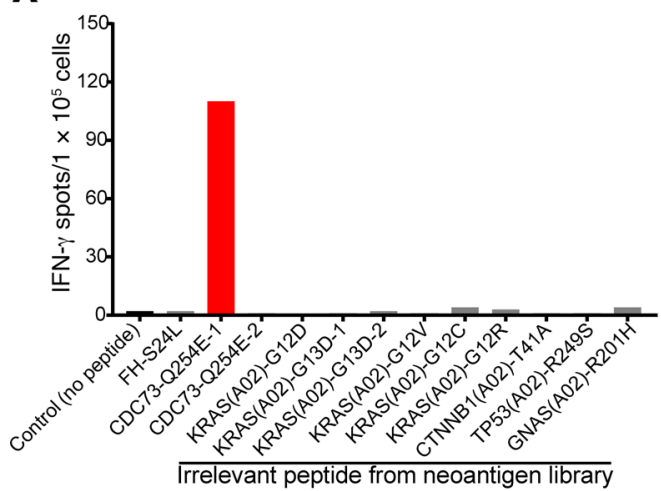

B
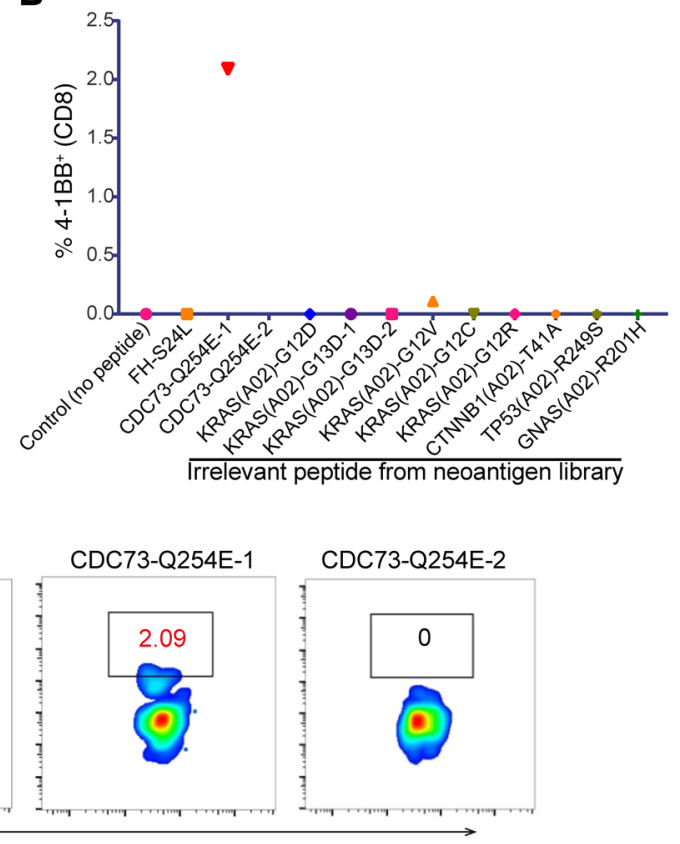

D

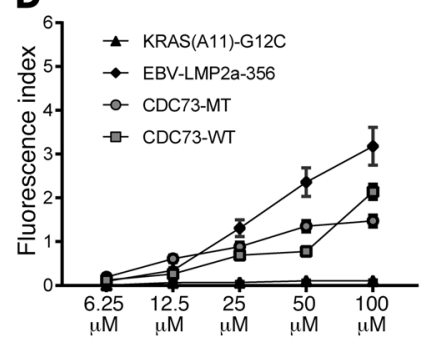

E

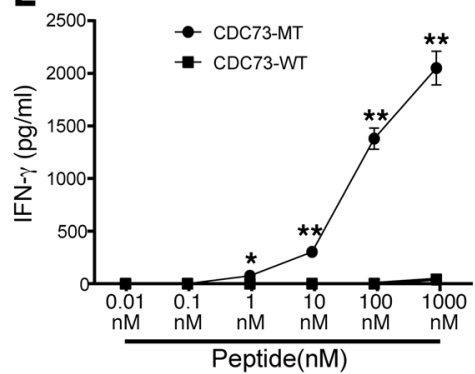

$\mathbf{F}$

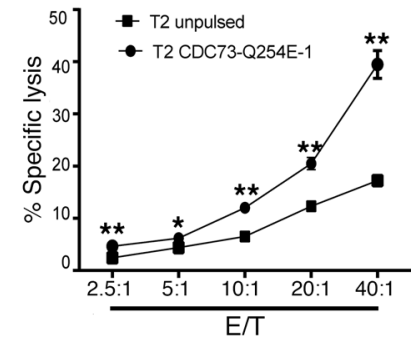

Figure 2. Characterization and immunogenicity testing of neoantigen in patient A017 with metastatic thymoma. (A-C) Three HLA-A*0201-restricted candidate mutant peptides and 9 irrelevant mutant peptides from the shared neoepitope peptide library were selected to assess the $T$ cell-specific antigen response. After 10-day recall memory T cell assay, IFN- $\gamma$ ELISPOT (A) and flow cytometry (B and C) were performed to measure the IFN- $\gamma$ and 4-1BB expression (gated on CD3). (D) T2 cells were cocultured with the mutant CDC73 (CDC73-MT) and the corresponding wild-type (CDC73-WT) peptides to assess the binding affinity to HLA-A*0201. The HLA-A*0201-restricted CMV-pp65-495, EBV-LMP2a-356, and EBV-LMP2a-426 peptides were used as positive control; the HLA-A*1101-restricted KRAS-G12C peptide was used as negative control. The fluorescence index is shown for each peptide. (E) IFN- $\gamma$ release measured by cytometric bead array after overnight coculture of T cells with $\mathrm{T} 2$ cells that were pulsed with the indicated concentrations of mutant peptides and corresponding wild-type peptides. (F) NRTs (bulk T cells) were cocultured with CFSE-labeled T2 cells that were pulsed with mutant CDC73 peptide or T2 cells not pulsed with peptide at an effector/target (E/T) ratio of 2.5:1, 5:1, 10:1, 20:1, and 40:1, respectively. After 6 hours, propidium iodide (PI) was added and the $\mathrm{PI}^{+} \mathrm{CFSE}{ }^{+} \mathrm{T}$ cells were analyzed by FACS. A-C are representative of 3 independent experiments. In $\mathbf{D}-\mathbf{F}$, data are presented as mean $\pm \operatorname{SEM}(n=3) ;{ }^{*} P<0.05,{ }^{*} P<0.01$ by 2 -tailed Student's $t$ test.

Shared neoantigen peptide library-guided neoantigen identification. In clinic, a large number of patients with refractory advanced solid tumor underwent targeted sequencing, aimed mainly at seeking targeted drugs, with the use of different types of gene panels, including 416 genes, 112 genes, and 382 genes. Among these patients, immunogenic neoantigen identification was performed, by detection of the secretion of IFN- $\gamma$ using ELISPOT and cytometric bead array, on 13 patients who harbored corresponding hotspot mutations and common HLA-A alleles matched to the shared neoantigen peptide library. Immunogenic neoantigens recognized by autologous PBMCs based on neoantigen peptide library were identified in 6 patients (Supplemental Table 12).

Neoantigen-based clinical translational immunotherapy research. Six patients with relapsed and refractory solid tumors origina- ting from the pancreas, thymus, or uterus, who had successfully identified neoantigens by 2 different pipelines, received personalized immunotherapy targeting 1 dominant neoepitope. Each patient received no fewer than 2 cycles of treatment. Approximately $1 \times 10^{7}$ neoantigen-loaded DCs and $1 \times 10^{10}$ bulk $\mathrm{T}$ cells composed of $1 \times 10^{9}$ neoantigen-reactive $\mathrm{CD} 8^{+} \mathrm{CD} 137^{+}$ $\mathrm{T}$ cells (NRTs) were generated for personalized immunotherapy in each cycle. Phenotypes were tested before the immunotherapy, including the composition of the transferred cell populations (NK, $\mathrm{B}, \mathrm{CD}^{+}$, and $\mathrm{CD}^{+} \mathrm{T}$ cells), expression of costimulatory molecules of T cells (CD27, CD28, PD-1, TIM-3, LAG-3), CD137 expression level, and in vitro antigen-specific killing (Supplemental Table 13 and Supplemental Figures 1 and 3). The NRTs among the adoptively transferred cells could specifically lyse T2/T2-A11 target 
A

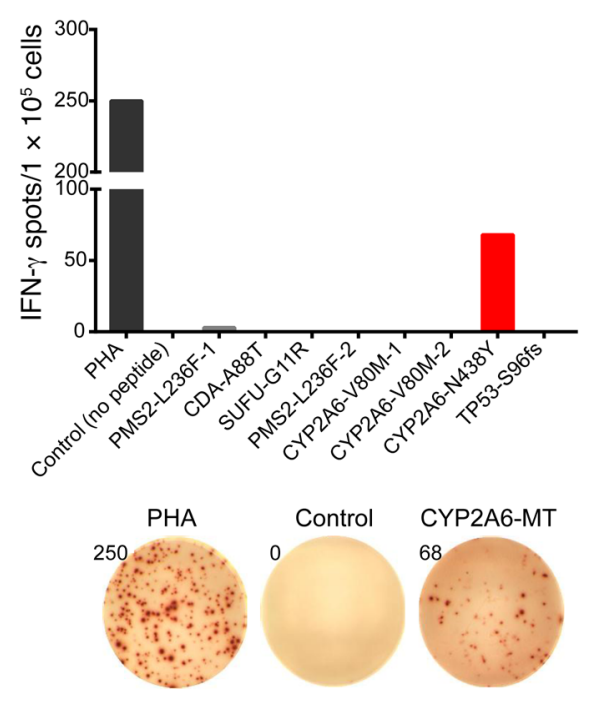

B

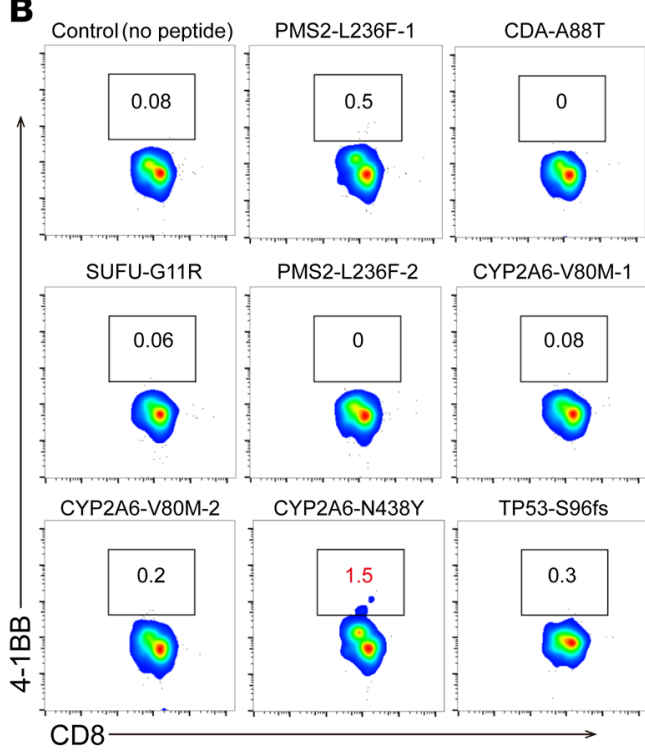

Figure 3. Identification of personalized neoantigen in patient $\mathrm{AOO} 4$ with advanced gastric cancer. (A) Autologous PBMCs were stimulated with 8 candidate mutant peptides for 10 days, after which IFN- $\gamma$ ELISPOT assays were performed to assess the T cell-specific antigen response. (B) FACS was used to detect 4-1BB upregulation on $\mathrm{CD}^{+} \mathrm{T}$ cells (gated on CD3). Phytohemagglutinin (PHA) was used as positive control, and no-peptide stimulation was tested as negative control. Data are representative of 3 independent experiments. cells loading the corresponding mutant peptides, especially the enriched NRTs after sorting and expansion (Supplemental Figure 3). High correlations of CD137 expression with intracellular cytokine staining data of IFN- $\gamma$ and TNF- $\alpha$ in enriched NRTs were found after corresponding mutant peptide stimulus (Supplemental Figure 4). In addition, flow cytometry analysis of memory or activation markers showed that the majority of infused NRTs were the central memory phenotype $\left(\mathrm{CD} 45 \mathrm{RO}^{+} \mathrm{CD} 62 \mathrm{~L}^{+}\right)$and naive phenotype (CD45RO-CD62 $\mathrm{L}^{+}$) (Supplemental Figure 5), which exhibited superior antitumor activity and superior survival (23). Before vaccination and $\mathrm{T}$ cell reinfusion, patients received radiotherapy or chemotherapy according to immunomodulatory strategies, which were designed to better exert synergistic antitumor effects in refractory solid tumors. (The clinical characteristics and treatment scheme are shown in Supplemental Tables 14 and 15.)

A 52-year-old man (ID: A017) with multiple metastatic tumor nodules in the left lower lung after the resection of thymoma was resistant to 3 lines of radiotherapy and chemotherapy, and CT chest scans revealed an increase in the number of tumor nodules within the left lung. He was subsequently enrolled in and underwent 5 cycles of personalized immunotherapy targeting somatic CDC73-Q254E mutation (Figure 6A). CT scans performed after 6 cycles revealed complete remission (CR) of all metastatic tumor nodules, and CR had lasted beyond 29 months to date (Figure 6B). Month 6 after treatment, PBMCs showed a stronger response to the mutant CDC73-Q254E peptide in contrast to before treatment (Figure 6C), and a striking increase in T cells specific for some tumor-associated antigens was also observed, such as AGR2, SART3, NY-ESO-1, and WT-1 (Figure 6, D and E, and Supplemental Table 16). In contrast, such obvious epitope spreading was not demonstrated in the other 5 patients.

In addition, a 35-year-old woman with metastatic pancreatic cancer (ID: C003) received 4 cycles of personalized neoantigen-based immunotherapy targeting HLA-A $^{*}$ 0201-restricted KRAS-G12D epitope (Figure 7, A and B). The PET-CT scan performed 2.5 months after immunotherapy showed a remarkable regression of multiple retroperitoneal and mediastinal metastatic lesions, whereas a few metastatic lesions remained refractory (Figure 7C), and this patient had a 2.9-month immune-related partial response (irPR; according to irRECIST). The remaining 4 patients achieved prolonged stable disease with median progression-free survival of 8.6 months (Supplemental Table 15). Grade 1 and grade 2 side effects, such as fever, chills, vomiting, and local temporary rash at the vaccine site, were observed during immunization; no serious adverse events were noted in any patient (Supplemental Table 17).

\section{Discussion}

Identification of individualized immunogenic neoepitopes is the major obstacle to translating clinical studies into neoantigenbased cancer immunotherapy. In this study, 2 different patterns of screening of neoantigen were established and successfully applied to personalized immunotherapy for patients with refractory advanced solid tumors.

A targeted sequencing-based de novo peptide synthesis pattern was set up as the first model. With this pattern, immunogenic neoantigens could be recognized by autologous T cells in 3 of 4 patients. Two of 9 (ID: A008), 1 of 3 (ID: A017), and 1 of 8 (ID: A004) candidate mutant peptides induced significant peptide-specific $\mathrm{T}$ cell responses. For the 3 patients, fewer than 10 candidate peptides were synthesized; subsequently, 1-2 neoantigens were identified by autologous $\mathrm{T}$ cells. As previous studies reported, only 1-3 neoantigens of around 50,153 , and 501 candidate peptides could be recognized by tumor-infiltrating lymphocytes $(9,24,25)$. The number of neoantigens identified in this study was slightly lower compared with the previous reports, but the number of candidate peptides was reduced significantly.

The following designs and methods in contrast to previous studies improved the feasibility and efficiency of neoantigen screening: Firstly, both the variant allele frequency and the MHC-peptide binding affinity were evaluated to optimize candidate epitopes; thereby the range of candidate mutations and predicted peptides was narrowed. Rosenberg's team identified 
A

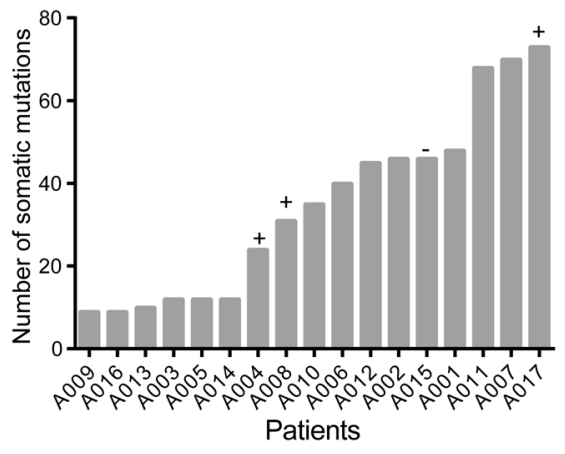

B

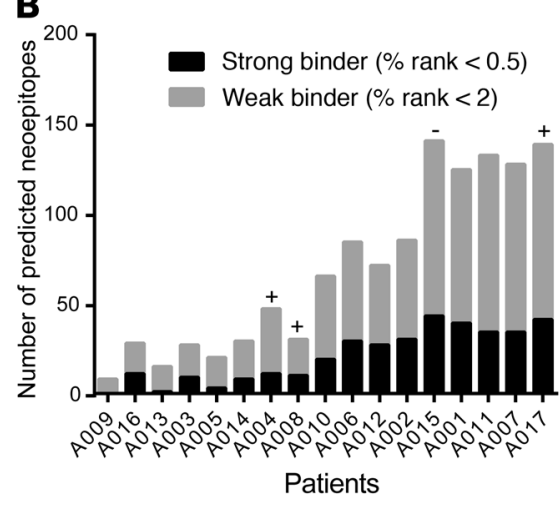

Figure 4. Frequency of somatic mutations and predicted epitopes in 17 patients with advanced solid tumor. (A) A large clinical-grade targeted sequencing panel of 416 cancer-related genes was performed in 17 patients with advanced solid cancer. Tumor-specific somatic mutations were identified. The frequency of somatic missense mutations of each patient is shown. (B) The frequency of neoantigen epitopes was predicted for each patient's nonsynonymous single-nucleotide variations of the restricting HLA class I alleles (HLA-A, HLA-B, and HLA-C). "+" indicates screened tumor samples in which neoantigen-specific T cell responses were detected; "-" indicates the 1 screened tumor sample in which no neoantigen-specific T cell response was detected.

neoantigens in 9 of 10 patients with gastrointestinal tumor with high-throughput immunological screening of TMGs, and revealed that all the identified $\mathrm{CD} 8^{+} \mathrm{T}$ cell epitopes were predicted to rank among the top 2\% of peptides with high MHC-peptide binding affinity (26). These data support the design of the present study to narrow the number of candidate peptides. The variation detected by targeted sequencing was less than that detected by WES; thus, multiple approaches were applied to detect mutations to avoid the omission of important variation information. Meanwhile, as the immunogenic mutations exhibited a very wide range of expression levels (2.9-185.4 fragments per kilobase of transcript per million mapped reads) (26), the low-frequency mutation with an AF lower than the $2 \%$ threshold was simply removed. Secondly, the in vitro neoantigen identification method was based on a 10-dayrecall memory $\mathrm{T}$ cell response. More recently, a similar method that selected somatic mutations with high variant allele frequency and identified immunogenic neoantigens was performed by 12-day-recall IFN- $\gamma$ ELISPOT assay using PBMCs, and 1 or 2 neoantigens were validated per patient out of 8-9 candidate peptides (27); this was consistent with the present study. The manner and time in which the $\mathrm{T}$ cells were stimulated by antigens in vitro are a key factor affecting the immune responses. For instance, $\mathrm{T}$ cell responses observed after several approximately 3 -week rounds of stimulations based on artificial APCs or irradiated APCs were confirmed to be mediated by de novo-primed naive $\mathrm{T}$ cells rather than by preexisting memory $\mathrm{T}$ cells, as short-time stimulation of the same PBMCs did not result in the detection of specific $\mathrm{T}$ cell clones (28-30). In this study, the neoantigen identification was based on the detection of spontaneous memory $\mathrm{T}$ cell responses, which may be more suitable for refractory advanced solid tumors with very short survival time: (a) A therapeutic vaccine for preexisting antigen-specific $\mathrm{T}$ cells, which were produced as a secondary immune response after vaccination, was more rapid and more intense. Meanwhile, the expansion of NRTs was also more rapid. (b) The detection of preexisting memory $\mathrm{T}$ cells may represent that the specific spontaneous antigen processingpresentation-recognition pathway was complete.

Mutations identified in targeted sequencing panels that query a subset of cancer-related genes have been widely implicated in cancer biology or clinical management (21, 31-34). Furthermore, large targeted sequencing panels $(n>300)$ can be used to assess the mutational load and the efficacy of anti-programmed cell death protein 1 (anti-PD-1) therapy $(35,36)$. The assessment of the use of a targeted panel of 416 genes in 17 patients with refractory advanced solid tumors in this study also indicated that targeted sequencing could mine enough mutations for personalized neoantigen identification. However, small panels had little genetic variation in clinical detection, and the potential for identifying personalized neoantigens was limited. An immunogenic mutation (CYP2A6-N438Y, KRYCFGEGL) was successfully identified in the ctDNA sample of patient A004 using the targeted sequencing panel. Indeed, it is not always possible to obtain tumor tissue samples for direct genomic analysis; therefore, it is more meaningful to detect the relevant indicators in the blood ("liquid biopsy"). The ctDNA from the various parts of the tumor was released into the blood, which could better reflect the patient's overall tumor burden, malignancy, metastatic capacity, and real-time gene mutation information. However, ctDNA represents from less than $0.1 \%$ to $10 \%$ of the total circulating free DNA (cfDNA) in plasma and serum (37-39); thus extremely high-depth sequencing is required to effectively detect tumor-derived genome mutations. Researchers often increase the depth of targeted sequencing to more than $5000 \times$ to $30,000 \times$, to call somatic variants in the cfDNA sample $(40,41)$. The WES and transcriptome sequencing techniques, which were commonly used in the identification of neoantigens, are difficult to apply to "liquid biopsy" because of their limited sequencing depth (always at 100x to 200x), whereas the targeted sequencing might mitigate this challenge.

An inventory-shared neoantigen peptide library was constructed as the second model, which aimed to identify neoantigens in a timely and convenient manner. The HLA- $A^{*} 02$, HLA-A ${ }^{*} 24$, and HLA-A ${ }^{*} 11$ alleles, selected for neoantigen peptide library construction, were collectively expressed in $44 \%$, $30 \%$, and $13 \%$ of the White population, respectively, and also expressed in up to approximately $37.7 \%, 31.6 \%$, and $61 \%$ of the Chinese population, respectively (42). Moreover, the 29 selected hotspot mutations covered $9.49 \%-89.56 \%$ patients in the 9 


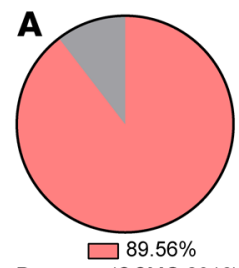

Pancreas (QCMG 2016)

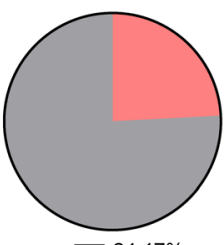

$\square 24.17 \%$
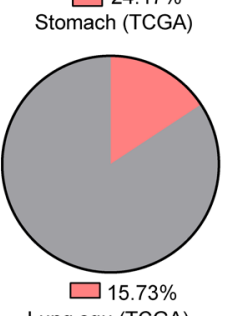

Lung squ (TCGA)
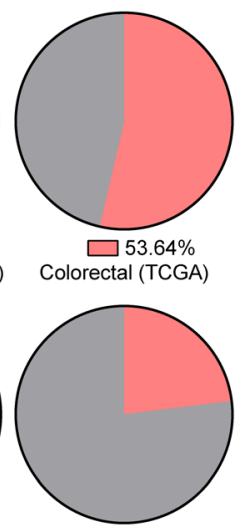

$\square 23.04 \%$

Cervical (TCGA)

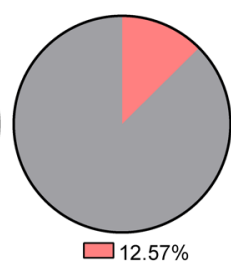

Liver (TCGA)

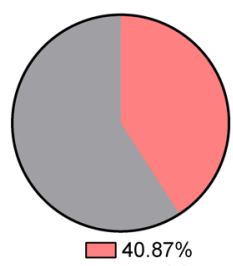

Lung adeno (TCGA)

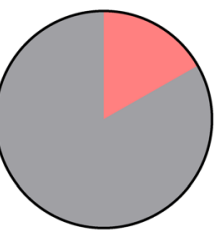

$\square 16.77 \%$
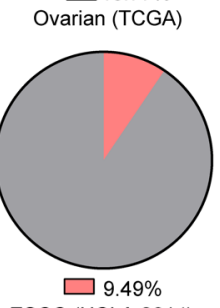

ESCC (UCLA 2014)

$\square$ Coverage of hotspot mutations
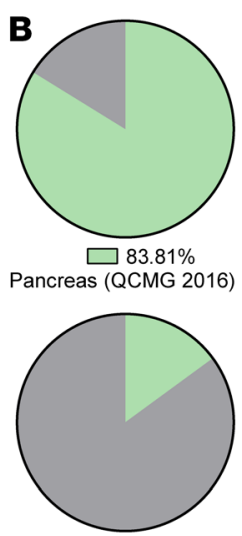

$\square 14.94 \%$

Stomach (TCGA)

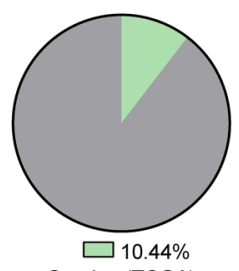

Ovarian (TCGA)
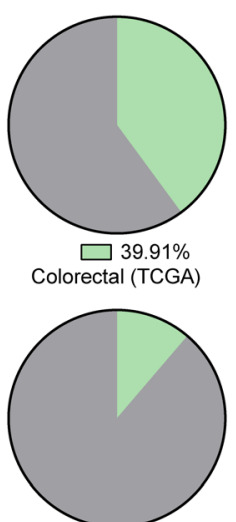

$\square 11.26 \%$

Liver (TCGA)

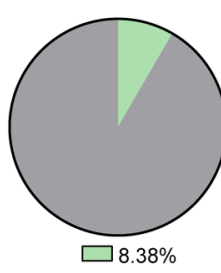

Lung squ (TCGA)

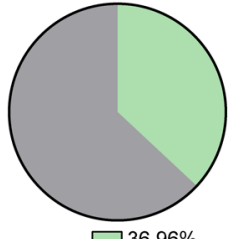

Lung adeno (TCGA)

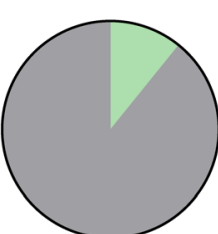

$\square 10.82 \%$

Cervical (TCGA)

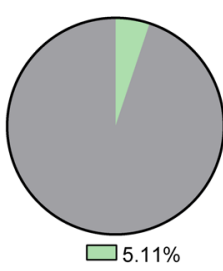

ESCC (UCLA 2014)

$\square$ Coverage of neoantigen peptide library

Figure 5. The proportion of patients covered by the selected 29 hotspot mutations and the shared neoantigen peptide library (TCGA). (A)The proportion of cancer patients harboring the selected 29 hotspots in the TCGA database (9.49\%-89.56\%). (B)The proportion of patients in the TCGA database covered by the shared neoantigen peptide library (5.11\%-83.8\%). types of common solid tumors. Therefore, the shared neoantigen peptide library represents a large population of patients with tumor and has huge prospects for application. Clinical targeted sequencing and accurate typing of HLA took only 1 week; subsequently, neoantigen identification was performed immediately while patients' detected hotspot mutations and HLA alleles were matched to the off-shelf peptide library. This pattern significantly shortened the time compared with the de novo antigen synthesis and identification mode. More recently, a personalized neoantigen vaccine clinical trial indicated that a median time of 103 days was required from selection of mutations to RNA vaccine release, which was already the most rapid approach reported (43). In contrast, with an off-shelf neoantigen peptide library approach, immunogenic neoantigens recognized by autologous peripheral blood lymphocytes were successfully identified in 6 of 13 patients within 20 days. However, the harboring of hotspot mutations and common HLA alleles matched to the inventory-shared neoantigen peptide library was the limitation of this approach. The shared neoantigen peptide library is constantly updated and expanded, and the majority of mutant peptides have been confirmed as immunogenic epitopes in the ongoing clinical trials of personalized neoantigen-based immunotherapy (Chinese Clinical Trials Registry numbers ChiCTR-OIC-16010092, ChiCTR-OOC-16010023, ChiCTR-OIC-16010025, ChiCTR-OIC-17011275, and ChiCTROIC-17011913).

Effective antitumor immunity involves a series of stepwise events. The priming and activation of T cells mediated by DC vaccines and the recognition and killing of cancer cells by NRTs were only 2 steps in the cancer-immunity cycle; the combination of strategies that target other steps of the cycle may be more effective (44). In the present study, neoantigen-pulsed DC vaccines and NRTs were generated for personalized immunotherapy following immunomodulatory chemotherapy or radiotherapy. The chemo- therapy regimen of cyclophosphamide and gemcitabine can effectively reduce the number of inhibitory immune cells such as Tregs and myeloid-derived suppressor cells (MDSCs), and enhance the efficacy of immunotherapy (45-48). Low-dose irradiation programs tumor-associated macrophage differentiation to an iNOS ${ }^{+} /$ M1 phenotype that orchestrates effective $\mathrm{T}$ cell immunotherapy (49). Large-dose radiotherapy can increase the exposure of MHC class I molecules and new peptides on the tumor cell surface, and enhance antigen presentation as well as recognition of irradiated tumor by cytotoxic T lymphocytes (CTLs) (50). A previous work also showed that radiotherapy could promote the recruitment of activated $\mathrm{CD}^{+}$or $\mathrm{CD} 8^{+} \mathrm{T}$ cells to sites of inflammation by inducing the expression of CXCL16, CXCL10, and CCL5 chemokines in tumor cells, and then break tumor immune barriers that lead to the inhibition of tumor growth (51-53). The cytotoxic effect of radiotherapy may also break the preexisting and ongoing cellular immune response; therefore, the dosage, division mode, and timing of intervention required individualized cautious formulation. In the present study, the patients with locally advanced unresectable solid tumor received stereotactic body radiotherapy with a total dose of 40-60 Gy during the first immunotherapy cycle. For patients with metastases, partial lesions received low-dose radiation (0.5 Gy twice daily for 2 days) before the infusion of NRTs in each cycle. However, different combinations of radiotherapy, chemotherapy, and immunotherapy are still the focus of research that needs to be further explored in preclinical and clinical studies.

More recently, 2 clinical trials on neoantigen peptide or RNA vaccination showed great potential for application in melanoma $(43,54)$. These studies indicated that neoepitope vaccines alone could prevent disease recurrence in high-risk patients without radiologically detectable lesions. In contrast, the patients at a late stage or with detectable lesions still experienced recurrence and progression after the vaccination (43, 
A

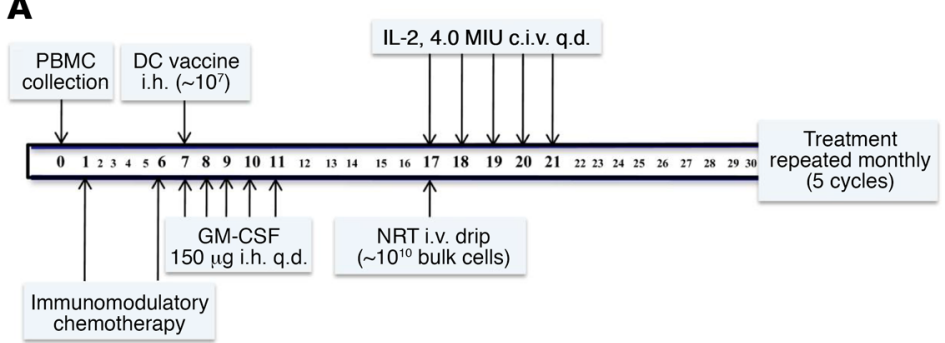

B
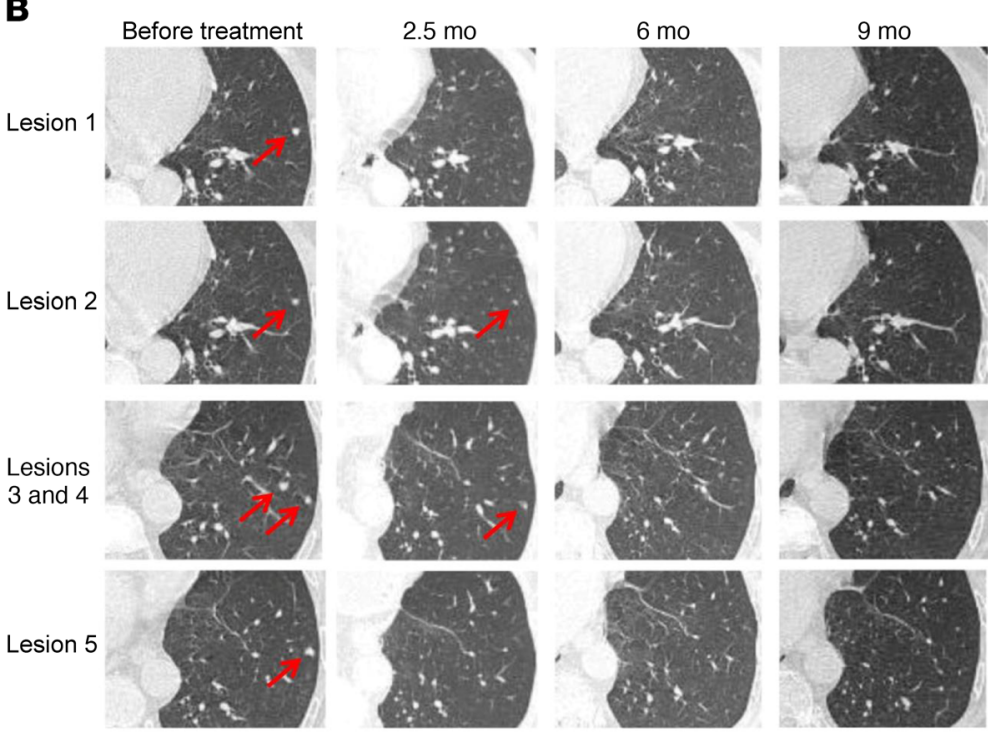

C

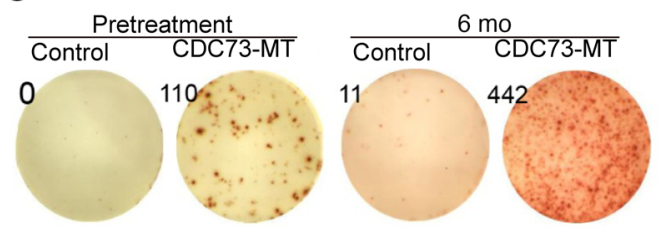

D

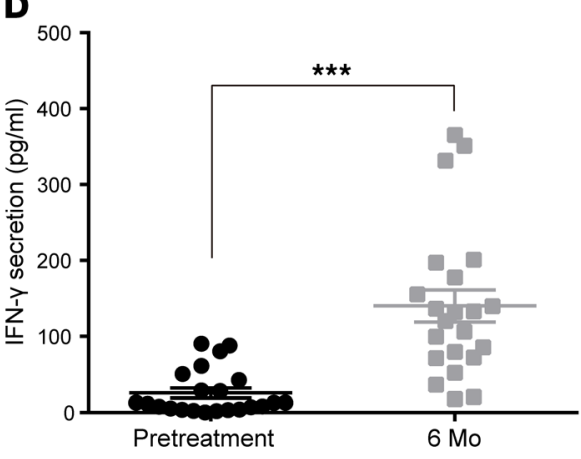

E

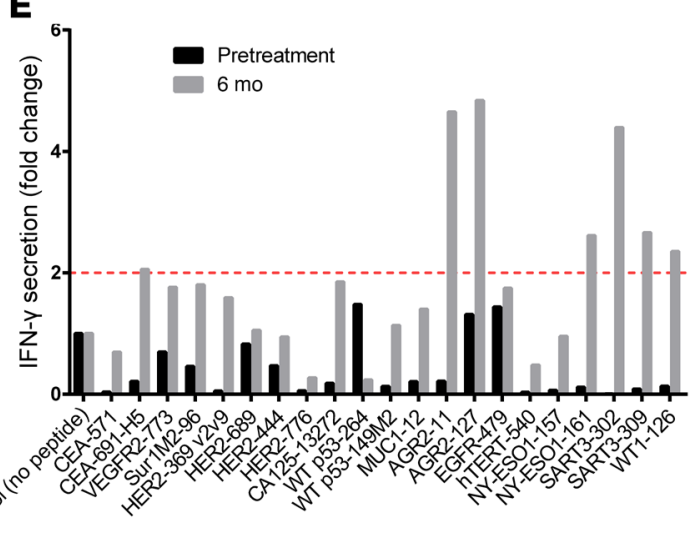

Figure 6. Immune and clinical responses to personalized immunotherapy in patient A017 with metastatic thymoma. (A) Treatment scheme: PBMCs were collected to generate neoantigen-loaded DC vaccines and NRTs in the laboratory. Before cell infusion, the patient was preconditioned with an immunomodulatory chemotherapy comprising $1000 \mathrm{mg} / \mathrm{m}^{2}$ gemcitabine on day 1 and day 6 and $250 \mathrm{mg} / \mathrm{m}^{2}$ cyclophosphamide on day 6 . Approximately $1 \times 10^{7}$ DC vaccines were inoculated (i.n.) subcutaneously on day 7, followed by subcutaneous injection of $150 \mu \mathrm{g}$ GM-CSF for 5 days. Approximately $1 \times 10^{10}$ bulk T cells composed of $1 \times 10^{9}$ NRTs were intravenously infused on day 17, followed by continuous intravenous (c.i.v.) injection of 4.0 million IU (MIU) IL-2 for 5 days. (B) CT scans were performed before and approximately 2.5 months, 6 months, and 9 months after personalized immunotherapy; representative radiological data are shown. (C) IFN- $\gamma$ ELISPOT showed changes in peptide-specific IFN- $\gamma$ secretion by patient PBMCs before and 6 months after treatment following 10-day culture with mutant CDC73 (CDC73-MT) or control. (D and E) Cytometric bead array assays demonstrated IFN- $\gamma$ secretion by PBMCs before and 6 months after treatment following 10-day culture with tumor-associated antigens and control. (D) ${ }^{* * *} P<0.001,2$-tailed Student's $t$ test, $n=3$. (E) Epitope spreading was demonstrated. Data from representative experiments are depicted $(n=3)$.

54). The increased tumor burden contributed not only to the increased tumor heterogeneity, but also to the decreased drug penetration, and the increased difficulty of lymphocyte infiltration (55). In the present clinical study, all the enrolled patients provided radiological or pathological evidence of detectable extensive metastasis or local progression. Since it was difficult to effectively control the disease with only the active immunization of tumor vaccine alone, active immunization of neoantigenloaded DC vaccines combined with passive immunization of NRTs and concurrent immunomodulatory chemotherapy or radiotherapy was adopted. POLE gene mutation was detected in the ctDNA sample of patient A017 with metastatic thymoma, who achieved a complete and durable response beyond 29 months, which was reported to represent an ultramutator phenotype beyond the MSI phenotype and an exceptional response to pembrolizumab in endometrial cancer (21). This suggests the need for further clinical investigation with immunotherapy specifically targeting solid tumors with POLE mutations, which are expected to be another marker to evaluate immunotherapy efficacy. Another patient, $\mathrm{C003}$ with metastatic pancreatic cancer, achieved a transient immune-related partial response (irPR). The median progression-free survival reached 8.6 months without serious adverse events, meaning a significant improvement in prognosis for these patients with refractory tumors.

In this study, personalized immunotherapy was not associated with PD-1/PD-L1 and other immune checkpoint inhibitors. The combination of neoantigen-specific immunotherapy and 
A

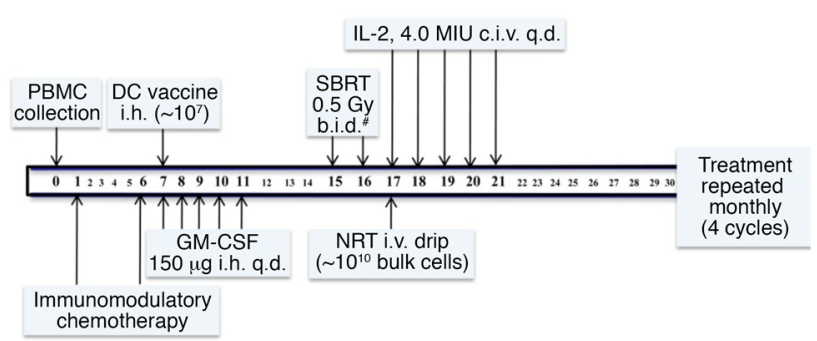

c

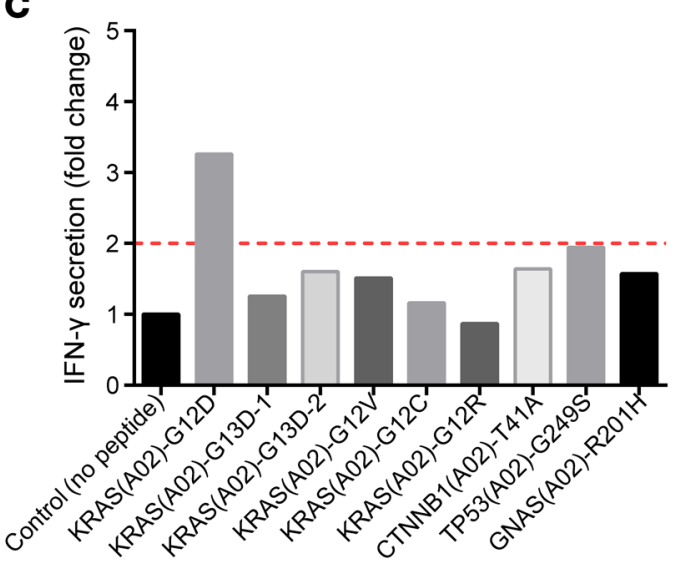

B

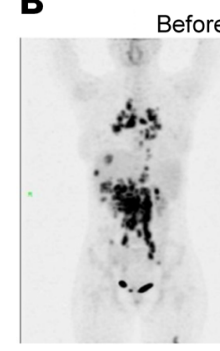

Before treatment

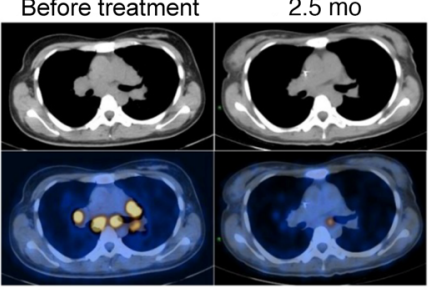

Before treatment

$2.5 \mathrm{mo}$

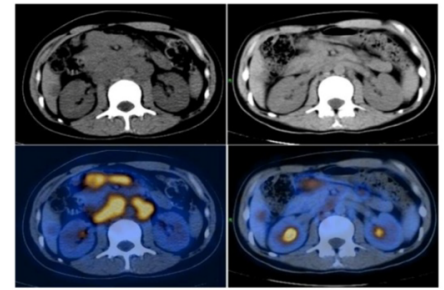

$2.5 \mathrm{mo}$
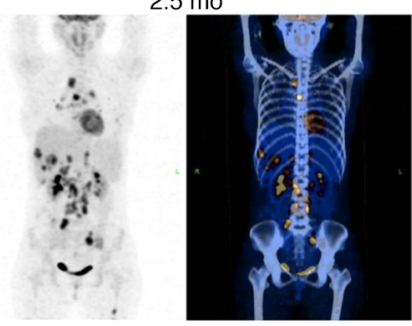

$2.5 \mathrm{mo}$

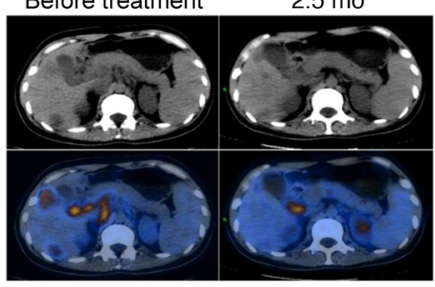

Before treatment $\quad 2.5 \mathrm{mo}$

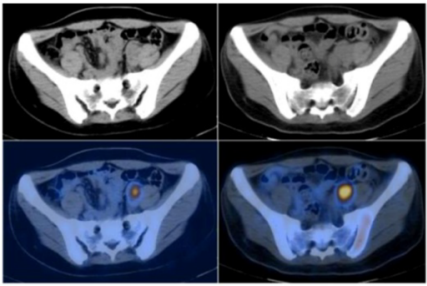

Figure 7. Tumor regression after treatment with KRAS-G12D-based personalized immunotherapy in patient C003. (A) Treatment scheme: PBMCs were collected to generate neoantigen-loaded DC vaccines and NRTs in the laboratory. Before vaccination, the patient was preconditioned with an immunomodulatory chemotherapy comprising $1000 \mathrm{mg} / \mathrm{m}^{2}$ gemcitabine on day 1 and day 6 and $250 \mathrm{mg} / \mathrm{m}^{2}$ cyclophosphamide on day 6 . DC vaccines were inoculated subcutaneously on day 7, followed by subcutaneous injection of $150 \mu \mathrm{g}$ GM-CSF for 5 days. Before NRT infusion, partial lesions received low-dose radiation (0.5 Gy twice daily for 2 days [\#]); NRTs were administered on day 17, followed by c.i.v. infusion of 4.0 MIU IL-2 for 5 days. (B) PET-CT scans were performed before and approximately 2.5 months after treatment; representative images are shown. (C) Representative data of immunogenic neoepitope identification using shared neoantigen peptide library.

checkpoint blockade may produce enhanced synergistic antitumor effects. Based on a prior work, which demonstrated that CRISPR/ Cas9-mediated PD-1 gene knockout could significantly increase the antitumor activity of EBV-specific CTLs in vitro and in vivo (56), two clinical trials have been initiated to evaluate PD-1-knockout EBV-CTLs for advanced-stage EBV-associated malignancies, as well as NRTs combined with PD-1 antibodies for Chinese patients with advanced refractory solid tumors (ClinicalTrials.gov NCT03044743 and NCT03171220, respectively). In addition, the adoptive transfer immunotherapy used in the present study targeted only 1 dominant neoepitope; the poly-neoepitopic immunity may reduce the risk of outgrowth of single neoantigen loss variants (57). Besides, $\mathrm{T}$ cell receptor engineering can also be considered to increase the proportion of mutation-specific $\mathrm{T}$ cells derived from peripheral blood lymphocytes in sufficient quantities for adoptive cell therapy. The inventory-shared neoantigen peptide library provides an opportunity to develop shared $\mathrm{T}$ cell receptor libraries against driver hotspot mutations in common solid tumors.

In summary, this study demonstrates a system combining targeted sequencing and a shared neoantigen peptide library and provides a pattern for timely and efficient identification of neoantigen, potentially paving the way to developing precision immunotherapeutic strategies with broad applicability for multiple malignant solid tumors.

\section{Methods}

Supplemental Methods are available online with this article; https:// doi.org/10.1172/JCI99538DS1.

Targeted next-generation sequencing. Between September 2014 and September 2017, a cohort of 27 patients with advanced solid tumors in Drum Tower Hospital, Medical School of Nanjing University, were undergoing tumor biopsies or blood withdrawal, including formalinfixed paraffin-embedded (FFPE) samples, biopsy specimens, serum samples, and serous effusions. Collected samples were sent to the core facility of Geneseeq Technology Inc. (Nanjing, China) for targeted next-generation sequencing (NGS) analysis. In brief, after sample preparation, DNA extraction, and library preparation, the enriched libraries were sequenced on HiSeq 4000 NGS platforms (Illumina) with coverage depths of at least $100 \times, 300 \times$, and 3000× after removal of PCR duplicates for blood, FFPE/pleural effusion, and ctDNA, respectively (58).

Trimmomatic was used for sequencing data quality control (59). The sequence reads with a quality below the threshold of 15 , as well as those with $\mathrm{N}$ bases removed, were mapped on the human reference sequence hg19 (Human Genome version 19) using Burrows-Wheeler Aligner software (BWA) (60). SNPs/indels were detected using Genome Analysis Toolkit (GATK)(61) and VarScan2 (62). SNPs were filtered out with dbSNP and 1000 Genome data sets. Germline mutations in tumor tissues or ctDNA were identified by comparing with 
the matched whole-blood DNA. Mutations were called when at least 3 mutated reads were found in the sample on different strands with good quality scores and manually inspected in Integrative Genomics Viewer (IGV, Broad Institute). Genomic fusions were identified by FACTERA (63) with default parameters. Whole NGS data were deposited in the NCBI's Sequence Read Archive database (accession no. SRP186418).

HLA typing. Four-digit HLA class I alleles (HLA-A, HLA-B, and HLA-C) and class II alleles (HLA-DRB1, HLA-DQB1) were identified by PCR-sequence-based typing (PCR-SBT) on patient peripheral blood (BGI, Shenzhen, China).

Epitope prediction and peptide synthesis. For each nonsynonymous mutation identified by targeted NGS, long peptides with 19 amino acids containing the mutated amino acid at position 10 were queried using the NetMHC 3.4/NetMHC 4.0 and NetMHCpan 3.0 tools to predict MHC class I binding of 8- to 10-mer mutant peptides to the patients' HLA-A, HLA-B, and HLA-C alleles (64-68). In addition, long peptides with 27 amino acids containing the mutated amino acid at position 14 were scanned to identify candidate 15 -mer peptides that were predicted to bind with high affinity to individual HLA class I alleles (HLA-DRB1) using the Immune Epitope Database (IEDB) and NetMHCII 2.2 analysis resource $(69,70)$. Peptides with an $\mathrm{IC}_{50}$ less than $500 \mathrm{nM}$ or \%rank less than 2.0 are predicted to be MHC binders. Peptides with $\mathrm{IC}_{50}$ less than $50 \mathrm{nM}$ or \% rank less than 0.5 are considered as strong binders. Customized peptides were obtained from ChinaPeptides and Bankpeptide yielding the same in vitro results.

Inventory-shared neoantigen peptide library construction. The TCGA and COSMIC databases were used to estimate the frequency of somatic missense mutations in human malignant solid tumors ( 71 , 72). The COSMIC database was used to assess the frequencies of each gene mutation in 9 types of common solid tumors - gastric cancer, colorectal cancer, esophageal squamous cell carcinoma, liver cancer, lung adenocarcinoma, lung squamous cell carcinoma, pancreatic cancer, ovarian cancer, and cervical cancer - as well as to calculate the frequencies of the specific point mutations in each gene by formula. In brief, genetic mutation data were downloaded from COSMIC (version 72, March 2015) and constantly updated. We obtained the frequency of genetic mutation $(A)$ by the formula $A=$ (number of mutated samples)/(number of samples tested) and calculated the proportion of the specific point mutation in each genetic mutation $(B)$ by the formula $B$ $=$ (number of instances of the specific point mutation) $/$ (total number of mutations). We calculated the frequency of specific point mutations $(F)$ in each cancer type by the formula $F=A \times B$ (73). Subsequently, we retrieved and analyzed the data from the TCGA data set by using cBioPortal (74) for Cancer Genomics to integrate the hotspots of all the missense mutations in 9 sequencing projects with the largest samples in the TCGA database.

The shared neoantigen epitopes were predicted by the hotspot mutations generated by in silico analysis of the TCGA and COSMIC databases. Identification of 8- to 10-mer peptides that were predicted to bind to human high-frequency HLA-A class I gene products HLA-A 02 ( $\left.A^{*} 0201, A^{*} 0203, A^{*} 0206\right), H_{L A} A^{*} 11$ (A*1101), and HLA-A 24 ( $\left.A^{\star} 2402\right)$ subtypes - was carried out using 19-mer peptides containing the mutated amino acid at position 10 with 5 programs using different algorithms: BIMAS (75), IEDB, NetMHC 3.4, NetCTL 1.2 (76), and SYFPEITHI (77). Based on the above analysis, 1 or 2 optimal specific HLA-restricted $\mathrm{T}$ cell epitopes were selected for each hotspot mutation. Neoantigen-derived peptides were synthesized, purified, and cryopreserved at $-80^{\circ} \mathrm{C}$ until used in a timely manner.

Analysis of $\mathrm{T}$ cell responses. Patients' autologous PBMCs were used to evaluate the immunogenicity of candidate neoantigens in vitro. An established simple and effective culture protocol with a few modifications was mainly used in detecting and monitoring antigen peptidespecific CTL precursors in the circulation as previously reported (78, 79). Briefly, heparinized blood samples were obtained from patients with relapsed/refractory tumor for the isolation of PBMCs by centrifugation on a Ficoll density gradient and suspended in AIM-V medium (Gibco). In each U-bottomed well, $1 \times 10^{5} \mathrm{PBMCs}$ were incubated with a corresponding peptide $(25 \mu \mathrm{M})$ in $200 \mu$ culture medium, which was applied to facilitate cell-to-cell contact. The culture medium consisted of AIM-V medium, 10\% FCS (Gibco), and IL-2 (100 U/ml; PeproTech). For peptide stimulation at 3-day intervals, half of the culture medium containing a corresponding peptide $(25 \mu \mathrm{M})$ and IL-2 $(100 \mathrm{U} / \mathrm{ml})$ was changed. After 3 cycles of peptide stimulation followed by an overnight restimulation, on day 10 , the specific $\mathrm{T}$ cell responses to each peptide were evaluated by ELISPOT. Recognition of the single antigens was tested as compared with no-peptide (media) control, and stimulus phytohemagglutinin was used as positive control. In addition, the $\mathrm{T}$ cell activation marker 4-1BB (CD137) was assessed by flow cytometry.

In some cases, evaluation of the reactivity of $\mathrm{T}$ cells was carried out by peptide pulsing of DCs cocultured with T cells. Mature DCs were pulsed with $10 \mu \mathrm{M}$ peptide for $4-6$ hours at $37^{\circ} \mathrm{C}$, washed with prewarmed $\mathrm{PBS}$, and then incubated with $\mathrm{T}$ cells at a stimulator/ effector ratio of 1:10 in complete AIM-V medium overnight. The soluble IFN- $\gamma$ released from T cells was measured by INF- $\gamma$ ELISPOT, and the $\mathrm{T}$ cell activation marker 4-1BB was assessed by flow cytometry.

IFN- $\gamma$ ELISPOT assay. IFN- $\gamma$ ELISPOT kit (Dakewei) was used to determine the frequency of cytokine-secreting T cells after overnight activation with peptide (80). In this study, a multiple culture protocol was used to analyze $\mathrm{T}$ cell response as above. Briefly, peptidestimulated PBMCs or DC-pulsed peptide coculture with T cells $\left(10^{5}\right.$ per well) were added to duplicate wells for 18-20 hours. The plates were washed before the addition of the diluted detection antibody (1:100 dilution) and then incubated for 1 hour in $37^{\circ} \mathrm{C}$. After washing of the plates, streptavidin-HRP (1:100 dilution) was added and incubated at $37^{\circ} \mathrm{C}$ for another 1 hour. 3-Amino-9-ethylcarbazole (AEC) solution mix was then added to each well, and the plates were left in the dark for about 15-25 minutes at room temperature before deionized water was added to stop development. Plates were scanned by ELISPOT CTL Reader (Cellular Technology Inc.), and the results were analyzed with ElisPot software (AID). Spots greater than twice the no-peptide (media) control were considered positive for $\mathrm{T}$ cell reactivity.

Cytometric bead array analysis of cytokines. The concentrations of cytokines in culture supernatants were measured by cytometric bead array according to the manufacturer's protocol (BD Biosciences) with an appropriate diluent. Human IFN- $\gamma$ Flex Set (Bead B8) (BD Biosciences) was used for detection of single-cytokine IFN- $\gamma$. The samples were run and FACS data were collected using an Accuri C6 (BD Biosciences) flow cytometer and analyzed using FCAP version 3.0 array software (Soft Flow).

Peptide binding assay. The HLA-A ${ }^{*} 0201-$ positive T2 cells (ATCC) with antigen-processing defects that allow for the efficient loading of exogenous peptides were used as an assay of candidate HLA-A ${ }^{*} 0201$ 
peptide binding efficiency $(81,82)$. Specifically, T2 cells were cultured for 24 hours in serum-free RPMI 1640 medium. Cells were then washed and resuspended in serum-free RPMI 1640 medium and plated to triplicate wells of a 96-well U-bottomed microtiter plate at $1 \times 10^{5}$ cells per well. Different dilutions of $100 \mu \mathrm{M}, 50 \mu \mathrm{M}, 25 \mu \mathrm{M}$, $12.5 \mu \mathrm{M}$, and $6.25 \mu \mathrm{M}$ of peptides and $5 \mu \mathrm{g} / \mathrm{ml}$ of human $\beta_{2}$-microglobulin (Sigma-Aldrich) were added to the culture medium for 16 hours at $37^{\circ} \mathrm{C}, 5 \% \mathrm{CO}_{2}$. After incubation, the cells were washed, and surface levels of HLA-A*0201 were assessed by staining with PE-conjugated mouse anti-human HLA-A2 monoclonal antibody (Medical \& Biological Laboratories) for 30 minutes at $4^{\circ} \mathrm{C}$ in the dark. The MHC-bound fluorescence level was measured by flow cytometry. The fluorescence index (FI) was calculated as follows: $\mathrm{FI}=$ (mean PE fluorescence with the given peptide - mean PE fluorescence without peptide)/(mean PE fluorescence without peptide).

Cytotoxicity assay. The neoantigen-specific CTLs were tested for lytic activities by CFSE/propidium iodide labeling cytotoxicity assay. T2/T2-A11 cells pulsed with corresponding peptides and T2/ T2-A11 cells only were used as target cells (the T2-A11 cells expressing HLA-A ${ }^{\star} 1101$ were constructed by our library). Target cells were labeled with $4 \mathrm{mM}$ carboxyfluorescein succinimidyl ester (CFSE; Invitrogen) for 10 minutes at $37^{\circ} \mathrm{C}$ in PBS. Labeling was stopped by addition of 10-fold volume of PBS and extensively washed in PBS before seeding into the 24-well plates. CFSE-labeled cells were then incubated with $\mathrm{T}$ cells at different effector/target ratios for 6 hours. Propidium iodide (Sigma-Aldrich) was added to determine the ratio of cell death. Samples were analyzed by flow cytometry.

Generation of DCs and neoantigen-specific T cells. PBMCs were collected with COBE Spectra MNC program (Terumo BCT). The in vitro cell processing and expansion were performed in a GMP-compliant laboratory. Monocyte-derived DCs were generated by plate adherence of PBMCs. Briefly, PBMCs were set to $5 \times 10^{6}$ to $10 \times 10^{6}$ cells $/ \mathrm{ml}$ in AIM-V medium and incubated for 2 hours at $37^{\circ} \mathrm{C}, 5 \% \mathrm{CO}_{2}$. Then, nonadherent cells were collected and washed. The adherent cells were cultured for 72 hours with CellGro DC media (CellGenix) containing 1\% human serum (HS; collected and processed in-house), GM-CSF (800 $\mathrm{IU} / \mathrm{ml})$, and IL-4 (1000 IU/ml). The immature DCs were then lifted and resuspended in fresh medium containing 1\% HS, GM-CSF (800 $\mathrm{IU} / \mathrm{ml})$, IL-4 (1000 IU/ml), LPS (10 ng/ml), and IFN- $\gamma(100 \mathrm{IU} / \mathrm{ml})$ (LPS from Sigma-Aldrich, cytokines from PeproTech) and incubated for approximately 16-48 hours. Flow cytometry was used to characterize the phenotype of the cells by the expression of CD11c, CD54, CD86, and HLA-DR (all from BD Biosciences) to ensure that the cells were predominantly mature DCs. Mature DCs were harvested and used to prepare DC vaccines and amplify antigen-specific T cells.

Mature DCs were pulsed with identified peptides $(10 \mu \mathrm{M})$ individually for approximately $4-6$ hours at $37^{\circ} \mathrm{C}$, washed with prewarmed PBS. Then, approximately $2 \times 10^{7}$ to $4 \times 10^{7}$ washed DCs were resuspended with normal saline (NS) to prepare DC vaccines. Peptide-pulsed DCs were incubated with $\mathrm{T}$ cells at a ratio of $1: 5$ to $1: 10$ in complete AIM-V medium supplemented with 5\% HS, IL-2 (100 U/ml), IL-7 (10 ng/ml), and IL-15 (10 ng/ $\mathrm{ml}$ ). The fresh complete medium containing cytokines was added every 2-3 days. On day 7 to 10 , the proportion of neoantigenspecific $\mathrm{T}$ cells was assessed by flow cytometry or ELISPOT assays. According to the growth of the neoantigen-specific $\mathrm{T}$ cells, the OKT3 antibody and the irradiated K562-based artificial APCs loading antigen were cocultured with $\mathrm{T}$ cells for restimulation. (The K562 cells expressing CD137L, CD80, and HLA-A*1101/ HLA-A ${ }^{*} 0201$ were constructed by our library.) Up to day 17 , the antigen-specific $\mathrm{T}$ cells were washed and resuspended with NS. Before cell transplantation, phenotypes were analyzed using flow cytometry, and quality control criteria were administered (endotoxin testing $\leq 5 \mathrm{EU} / \mathrm{ml}$, a negative result for mycoplasma and sterile detection) to confirm the asepsis of the products.

Cell sorting and expansion. The proportion of neoepitope-specific $\mathrm{T}$ cells in bulk $\mathrm{T}$ cultures used to treat patients was evaluated by fluorescent MHC tetramers. A previously described UV-mediated peptide exchange procedure was used to generate $A^{\star} 1101$-mutant peptide tetramers (83). Briefly, according to the procedure of the Flex-T MHC Tetramers Kit (BioLegend), HLAs loaded with UV-sensitive peptide monomers were subjected to long-wave $(366 \mathrm{~nm})$ UV light in the presence of $50 \mu \mathrm{M}$ mutant peptide on ice for 1 hour. The monomer was then tetramerized in the presence of fluorescent (PE) streptavidin and kept at $4^{\circ} \mathrm{C}$ for cell staining. T cells were isolated by a FACSAria cell sorter after incubation with anti-CD8 (APC) and tetramers (PE) for 60 minutes and collected in sterile PBS containing 50\% FCS. The $\mathrm{CD}^{+}{ }^{+}$tetramer ${ }^{+} \mathrm{T}$ cells were amplified to large numbers using a rapid amplification protocol with IL-2 (600 U/ml; PeproTech), OKT3 antibody (30 ng/ml; eBioscience), and irradiated K562-A11 cells (at a 5:1 ratio of feeder cells to sorted $\mathrm{T}$ cells).

Statistics. GraphPad Prism 5.0 (GraphPad Software) was used for all statistical analysis. Data samples were compared using a 2-tailed Student's $t$ test, and a $P$ value of less than 0.05 was considered significant.

Study approval. This study was conducted with the approval of the Ethics Committee of Nanjing Drum Tower Hospital. All experimental methods and clinical treatment were carried out in accordance with the approved guidelines. Patients with advanced solid tumor who failed 2 or more treatment regimens or had no effective standard treatment available were included in the study. All the patients signed a statement of informed consent for scientific research.

\section{Author contributions}

FC designed the study, conducted experiments and in silico analysis, and wrote the manuscript text and prepared figures and tables. ZZ was involved in study design, conducted experiments and clinical studies, and prepared figures. JD, JS, FM, SS, and QX were involved in experiments and provided protocols for research. JY assisted in manuscript preparation and prepared figures. ND, YY, QL, SZ, and SD assisted in conducting experiments and prepared figures and tables. QW and ZS assisted in bioinformatics analysis. $\mathrm{BL}$ and JW designed and coordinated research and verified results. All authors reviewed the manuscript.

\section{Acknowledgments}

This work was supported by grants from the National Key Research and Development Program of China (2017YFC1308900), the National Major Projects for Major New Drugs Innovation and Development (2018ZX09301048-003), the National Natural Science Foundation of China (81672367, 81572329, 81572601), and the Key Research and Development Program of Jiangsu Province (BE2017607). We thank all patients and their families who participated in this study. We thank Geneseeq Technology Inc. for supporting the in silico analysis of next-generation sequencing. 
Address correspondence to: Baorui Liu or Jia Wei, The Comprehensive Cancer Centre of Drum Tower Hospital, Medical School of Nanjing University and Clinical Cancer Institute of Nanjing University,
321 Zhongshan Road, Nanjing 210008, Jiangsu Province, China. Phone: 86.25.83105082; Email: baoruiliu@nju.edu.cn (BL). Phone: 86.25.83106666.61331; Email: weijia01627@hotmail.com (JW).
1. Rosenberg SA. Cell transfer immunotherapy for metastatic solid cancer - what clinicians need to know. Nat Rev Clin Oncol. 2011;8(10):577-585.

2. Rosenberg SA, et al. Durable complete responses in heavily pretreated patients with metastatic melanoma using T-cell transfer immunotherapy. Clin Cancer Res. 2011;17(13):4550-4557.

3. Rizvi NA, et al. Activity and safety of nivolumab, an anti-PD-1 immune checkpoint inhibitor, for patients with advanced, refractory squamous nonsmall-cell lung cancer (CheckMate 063): a phase 2, single-arm trial. Lancet Oncol. 2015;16(3):257-265.

4. Powles T, et al. MPDL3280A (anti-PD-L1) treatment leads to clinical activity in metastatic bladder cancer. Nature. 2014;515(7528):558-562.

5. Topalian SL, et al. Survival, durable tumor remission, and long-term safety in patients with advanced melanoma receiving nivolumab. J Clin Oncol. 2014;32(10):1020-1030.

6. Muro K, et al. Pembrolizumab for patients with PD-L1-positive advanced gastric cancer (KEYNOTE-012): a multicentre, open-label, phase $1 \mathrm{~b}$ trial. Lancet Oncol. 2016;17(6):717-726.

7. Le DT, et al. PD-1 blockade in tumors with mismatch-repair deficiency. $N$ Engl JMed. 2015;372(26):2509-2520.

8. Zacharakis $\mathrm{N}$, et al. Immune recognition of somatic mutations leading to complete durable regression in metastatic breast cancer. Nat Med. 2018;24(6):724-730.

9. Robbins PF, et al. Mining exomic sequencing data to identify mutated antigens recognized by adoptively transferred tumor-reactive T cells. Nat Med. 2013;19(6):747-752.

10. Gubin MM, et al. Checkpoint blockade cancer immunotherapy targets tumour-specific mutant antigens. Nature. 2014;515(7528):577-581.

11. Rizvi NA, et al. Cancer immunology. Mutational landscape determines sensitivity to PD-1 blockade in non-small cell lung cancer. Science. 2015;348(6230):124-128.

12. Van Allen EM, et al. Genomic correlates of response to CTLA-4 blockade in metastatic melanoma. Science. 2015;350(6257):207-211.

13. Lu YC, Robbins PF. Cancer immunotherapy targeting neoantigens. Semin Immunol. 2016;28(1):22-27.

14. Lu YC, et al. Mutated PPP1R3B is recognized by $\mathrm{T}$ cells used to treat a melanoma patient who experienced a durable complete tumor regression. J Immunol. 2013;190(12):6034-6042.

15. Kreiter S, et al. Mutant MHC class II epitopes drive therapeutic immune responses to cancer. Nature. 2015;520(7549):692-696.

16. Tran E, et al. Cancer immunotherapy based on mutation-specific $\mathrm{CD} 4^{+} \mathrm{T}$ cells in a patient with epithelial cancer. Science. 2014;344(6184):641-645.

17. Lu YC, et al. Efficient identification of mutated cancer antigens recognized by $\mathrm{T}$ cells associated with durable tumor regressions. Clin Cancer Res. 2014;20(13):3401-3410.

18. Bocchia $\mathrm{M}$, et al. Complete molecular response in
CML after p210 BCR-ABL1-derived peptide vaccination. Nat Rev Clin Oncol. 2010;7(10):600-603.

19. Schumacher T, et al. A vaccine targeting mutant IDH1 induces antitumour immunity. Nature. 2014;512(7514):324-327.

20. Tran E, et al. T-cell transfer therapy targeting mutant KRAS in cancer. $N$ Engl J Med. 2016;375(23):2255-2262.

21. Mehnert JM, et al. Immune activation and response to pembrolizumab in POLE-mutant endometrial cancer. J Clin Invest. 2016;126(6):2334-2340.

22. Vogelstein B, Papadopoulos N, Velculescu VE, Zhou S, Diaz LA, Kinzler KW. Cancer genome landscapes. Science. 2013;339(6127):1546-1558.

23. Sommermeyer D, et al. Chimeric antigen receptormodified T cells derived from defined $\mathrm{CD}^{+}$and $\mathrm{CD} 4^{+}$subsets confer superior antitumor reactivity in vivo. Leukemia. 2016;30(2):492-500.

24. Linnemann C, et al. High-throughput epitope discovery reveals frequent recognition of neoantigens by $\mathrm{CD}^{+} \mathrm{T}$ cells in human melanoma. Nat Med. 2015;21(1):81-85.

25. Verdegaal EM, et al. Neoantigen landscape dynamics during human melanoma-T cell interactions. Nature. 2016;536(7614):91-95.

26. Tran E, et al. Immunogenicity of somatic mutations in human gastrointestinal cancers. Science. 2015;350(6266):1387-1390.

27. Zhang $X$, et al. Breast cancer neoantigens can induce $\mathrm{CD} 8{ }^{+} \mathrm{T}$-cell responses and antitumor immunity. Cancer Immunol Res. 2017;5(7):516-523.

28. Peper JK, et al. HLA ligandomics identifies histone deacetylase 1 as target for ovarian cancer immunotherapy. Oncoimmunology. 2016;5(5):e1065369.

29. Nelde A, et al. HLA class I-restricted MYD88 L265P-derived peptides as specific targets for lymphoma immunotherapy. Oncoimmunology. 2017;6(3):e1219825.

30. Nielsen JS, et al. Toward personalized lymphoma immunotherapy: identification of common driver mutations recognized by patient $\mathrm{CD} 8^{+} \mathrm{T}$ cells. Clin Cancer Res. 2016;22(9):2226-2236.

31. Frampton GM, et al. Development and validation of a clinical cancer genomic profiling test based on massively parallel DNA sequencing. Nat Biotechnol. 2013;31(11):1023-1031.

32. Wagle N, et al. High-throughput detection of actionable genomic alterations in clinical tumor samples by targeted, massively parallel sequencing. Cancer Discov. 2012;2(1):82-93.

33. Chen K, et al. Clinical actionability enhanced through deep targeted sequencing of solid tumors. Clin Chem. 2015;61(3):544-553.

34. Sohal DP, et al. Prospective clinical study of precision oncology in solid tumors. J Natl Cancer Inst. 2015;108(3):djv332.

35. Garofalo A, et al. The impact of tumor profiling approaches and genomic data strategies for cancer precision medicine. Genome Med. 2016;8(1):79.
36. Johnson DB, et al. Targeted next generation sequencing identifies markers of response to PD-1 blockade. Cancer Immunol Res. 2016;4(11):959-967.

37. Gorgannezhad L, Umer M, Islam MN, Nguyen NT, Shiddiky MJA. Circulating tumor DNA and liquid biopsy: opportunities, challenges, and recent advances in detection technologies. $L a b$ Chip. 2018;18(8):1174-1196.

38. Butler TM, Spellman PT, Gray J. Circulatingtumor DNA as an early detection and diagnostic tool. Curr Opin Genet Dev. 2017;42:14-21.

39. Molina-Vila MA, et al. Liquid biopsy in nonsmall cell lung cancer. Front Med (Lausanne) 2016;3:69.

40. Thompson JC, et al. Detection of therapeutically targetable driver and resistance mutations in lung cancer patients by next-generation sequencing of cell-free circulating tumor DNA. Clin Cancer Res. 2016;22(23):5772-5782.

41. Wyatt AW, et al. Genomic alterations in cell-free DNA and enzalutamide resistance in castrationresistant prostate cancer. JAMA Oncol. 2016;2(12):1598-1606.

42. González-Galarza FF, et al. Allele frequency net 2015 update: new features for HLA epitopes, KIR and disease and HLA adverse drug reaction associations. Nucleic Acids Res. 2015;43(Database issue):D784-D788.

43. Ott PA, et al. An immunogenic personal neoantigen vaccine for patients with melanoma. Nature. 2017;547(7662):217-221.

44. Chen DS, Mellman I. Oncology meets immunology: the cancer-immunity cycle. Immunity. 2013;39(1):1-10.

45. Ghiringhelli F, et al. Metronomic cyclophosphamide regimen selectively depletes $\mathrm{CD} 4^{+} \mathrm{CD} 25^{+}$ regulatory $\mathrm{T}$ cells and restores $\mathrm{T}$ and $\mathrm{NK}$ effector functions in end stage cancer patients. Cancer Immunol Immunother. 2007;56(5):641-648.

46. Chen C, Chen Z, Chen D, Zhang B, Wang Z, Le H. Suppressive effects of gemcitabine plus cisplatin chemotherapy on regulatory $\mathrm{T}$ cells in nonsmall-cell lung cancer. J Int Med Res. 2015;43(2):180-187.

47. Gebremeskel S, et al. Natural killer T-cell immunotherapy in combination with chemotherapy-induced immunogenic cell death targets metastatic breast cancer. Cancer Immunol Res. 2017;5(12):1086-1097.

48. Ghansah T, et al. Dendritic cell immunotherapy combined with gemcitabine chemotherapy enhances survival in a murine model of pancreatic carcinoma. Cancer Immunol Immunother. 2013;62(6):1083-1091.

49. Klug F, et al. Low-dose irradiation programs macrophage differentiation to an iNOS + /M1 phenotype that orchestrates effective T cell immunotherapy. Cancer Cell. 2013;24(5):589-602.

50. Reits EA, et al. Radiation modulates the peptide repertoire, enhances MHC class I expression, and induces successful antitumor immunotherapy. 
JExp Med. 2006;203(5):1259-1271.

51. Matsumura S, et al. Radiation-induced CXCL16 release by breast cancer cells attracts effector T cells. J Immunol. 2008;181(5):3099-3107.

52. Demaria S, Coleman CN, Formenti SC. Radiotherapy: changing the game in immunotherapy. Trends Cancer. 2016;2(6):286-294.

53. Zheng W, et al. Combination of radiotherapy and vaccination overcomes checkpoint blockade resistance. Oncotarget. 2016;7(28):43039-43051.

54. Sahin U, et al. Personalized RNA mutanome vaccines mobilize poly-specific therapeutic immunity against cancer. Nature. 2017;547(7662):222-226.

55. Zahreddine H, Borden KL. Mechanisms and insights into drug resistance in cancer. Front Pharmacol. 2013;4:28.

56. Su S, et al. CRISPR-Cas9-mediated disruption of PD-1 on human $\mathrm{T}$ cells for adoptive cellular therapies of EBV positive gastric cancer. Oncoimmunology. 2017;6(1):e1249558.

57. Matsushita H, et al. Cancer exome analysis reveals a T-cell-dependent mechanism of cancer immunoediting. Nature. 2012;482(7385):400-404.

58. Jin Y, et al. Mutational profiling of non-small-cell lung cancer patients resistant to first-generation EGFR tyrosine kinase inhibitors using next generation sequencing. Oncotarget. 2016;7(38):61755-61763.

59. Bolger AM, Lohse M, Usadel B. Trimmomatic: a flexible trimmer for Illumina sequence data. Bioinformatics. 2014;30(15):2114-2120.

60. Li H, Durbin R. Fast and accurate short read alignment with Burrows-Wheeler transform. Bioinformatics. 2009;25(14):1754-1760.

61. DePristo MA, et al. A framework for variation discovery and genotyping using next-generation DNA sequencing data. Nat Genet. 2011;43(5):491-498.

62. Koboldt DC, et al. VarScan 2: somatic mutation and copy number alteration discovery in cancer by exome sequencing. Genome Res. 2012;22(3):568-576.
63. Newman AM, et al. FACTERA: a practical method for the discovery of genomic rearrangements at breakpoint resolution. Bioinformatics. 2014;30(23):3390-3393.

64. Nielsen M, et al. Reliable prediction of T-cell epitopes using neural networks with novel sequence representations. Protein Sci. 2003;12(5):1007-1017.

65. Lundegaard C, Lamberth K, Harndahl M, Buus S, Lund O, Nielsen M. NetMHC-3.0: accurate web accessible predictions of human, mouse and monkey MHC class I affinities for peptides of length 8-11. Nucleic Acids Res. 2008;36(Web Server issue):W509-W512.

66. Andreatta M, Nielsen M. Gapped sequence alignment using artificial neural networks: application to the MHC class I system. Bioinformatics. 2016;32(4):511-517.

67. Nielsen M, Andreatta M. NetMHCpan-3.0; improved prediction of binding to MHC class I molecules integrating information from multiple receptor and peptide length datasets. Genome Med. 2016;8(1):33.

68. Hoof I, et al. NetMHCpan, a method for MHC class I binding prediction beyond humans. Immunogenetics. 2009;61(1):1-13.

69. Nielsen M, Lund O. NN-align. An artificial neural network-based alignment algorithm for MHC class II peptide binding prediction. BMC Bioinformatics. 2009;10:296.

70. Nielsen M, Lundegaard C, Lund O. Prediction of MHC class II binding affinity using SMM-align, a novel stabilization matrix alignment method. BMC Bioinformatics. 2007;8:238.

71. Cancer Genome Atlas Research Network, et al. The Cancer Genome Atlas Pan-Cancer analysis project. Nat Genet. 2013;45(10):1113-1120.

72. Forbes SA, et al. COSMIC: exploring the world's knowledge of somatic mutations in human cancer. Nucleic Acids Res. 2015;43(Database issue):D805-D811.
73. Karasaki T, et al. Identification of individual cancer-specific somatic mutations for neoantigenbased immunotherapy of lung cancer. JThorac Oncol. 2016;11(3):324-333.

74. Gao J, et al. Integrative analysis of complex cancer genomics and clinical profiles using the cBioPortal. Sci Signal. 2013;6(269):pl1.

75. Parker KC, Bednarek MA, Coligan JE. Scheme for ranking potential HLA-A2 binding peptides based on independent binding of individual peptide side-chains. J Immunol. 1994;152(1):163-175.

76. Larsen MV, Lundegaard C, Lamberth K, Buus S, Lund O, Nielsen M. Large-scale validation of methods for cytotoxic T-lymphocyte epitope prediction. BMC Bioinformatics. 2007;8:424.

77. Rammensee H, Bachmann J, Emmerich NP, Bachor OA, Stevanović S. SYFPEITHI: database for MHC ligands and peptide motifs. Immunogenetics. 1999;50(3-4):213-219.

78. Hida N, Maeda Y, Katagiri K, Takasu H, Harada $\mathrm{M}$, Itoh K. A simple culture protocol to detect peptide-specific cytotoxic T lymphocyte precursors in the circulation. Cancer Immunol Immunother. 2002;51(4):219-228.

79. Noguchi M, Sasada T, Itoh K. Personalized peptide vaccination: a new approach for advanced cancer as therapeutic cancer vaccine. Cancer Immunol Immunother. 2013;62(5):919-929.

80. Su S, et al. CRISPR-Cas9 mediated efficient PD-1 disruption on human primary $\mathrm{T}$ cells from cancer patients. Sci Rep. 2016;6:20070.

81. Tang Y, et al. An altered peptide ligand for naïve cytotoxic T lymphocyte epitope of TRP-2(180188) enhanced immunogenicity. Cancer Immunol Immunother. 2007;56(3):319-329.

82. Olson BM, et al. HLA-A2-restricted T-cell epitopes specific for prostatic acid phosphatase. Cancer Immunol Immunother. 2010;59(6):943-953.

83. Cohen CJ, et al. Isolation of neoantigen-specific $\mathrm{T}$ cells from tumor and peripheral lymphocytes. JClin Invest. 2015;125(10):3981-3991. 